\title{
Linear differential equations and multiple zeta values. I. Zeta(2)
}

\author{
by
}

\section{Michał Zakrzewski and Henryk Żołądek (Warszawa)}

\begin{abstract}
Certain generating fuctions for multiple zeta values are expressed as values at some point of solutions of linear meromorphic differential equations. We apply asymptotic expansion methods (like the WKB method and the Stokes operators) to solutions of these equations. In this way we give a new proof of the Euler formula $\zeta(2)=\pi^{2} / 6$. In further papers we plan to apply this method to study some third order hypergeometric equation related to $\zeta(3)$.
\end{abstract}

1. Introduction. Let $a_{1}, \ldots, a_{k}$ be integers such that $a_{i} \geq 1, a_{k} \geq 2$. The quantity

$$
\zeta\left(a_{1}, \ldots, a_{k}\right):=\sum_{0<n_{1}<\cdots<n_{k}} \frac{1}{n_{1}^{a_{1}} \ldots n_{k}^{a_{k}}}
$$

is called a multiple $\zeta$-value (see [15]). We introduce the following generating function for zeta values:

$$
\begin{array}{r}
f_{a_{1}, \ldots, a_{k}}(x):=1-\zeta\left(a_{1}, \ldots, a_{k}\right) x^{a}+\zeta\left(a_{1}, \ldots, a_{k}, a_{1}, \ldots, a_{k}\right) x^{2 a} \\
-\zeta\left(a_{1}, \ldots, a_{k}, a_{1}, \ldots, a_{k}, a_{1}, \ldots, a_{k}\right) x^{3 a}+\cdots
\end{array}
$$

where $a=a_{1}+\cdots+a_{k}$. We distinguish the generating function associated with $\zeta(2)$, i.e.

$$
\begin{aligned}
f_{2}(x) & =1-\zeta(2) x^{2}+\zeta(2,2) x^{4}-\cdots=\prod\left(1-\frac{x^{2}}{n^{2}}\right) \\
& =\frac{1}{\Gamma(1+x) \Gamma(1-x)}=\frac{\sin \pi x}{\pi x} .
\end{aligned}
$$

Formula (1.3) allows to show that $\zeta(2 m)=\pi^{2 m} \times($ rational number) (see [18]).

2010 Mathematics Subject Classification: Primary 05C38, 15A15; Secondary 05A15, $15 \mathrm{~A} 18$.

Key words and phrases: zeta function, hypergeometric differential equation, WKB expansion. 
In [18] the following result was proved. Consider the following differential operators which act on functions $\theta(t ; x), t \in \mathbb{R}$ :

$$
\partial=\partial / \partial t, \quad R=(1-t) \partial, \quad Q=t \partial, \quad P=R Q^{a_{1}-1} R Q^{a_{2}-1} \ldots R Q^{a_{k}-1} .
$$

THEOREM 1.1 ([18]). The generating function (1.2) equals

$$
f_{a_{1}, \ldots, a_{k}}(x)=\varphi_{1}(1 ; x),
$$

where $\varphi_{1}(t ; x)$ is the solution to the differential equation

$$
P g+x^{a} g=0
$$

such that $\varphi_{1}(t ; x)$ is analytic in $t$ near $t=0$ and $\varphi_{1}(0 ; x)=1$.

Equation (1.5) associated with the generating function $f_{2}(x)$ is the hypergeometric equation (with the parameters $\alpha=x, \beta=-x$ and $\gamma=1$ )

$$
(1-t) \partial t \partial g+x^{2} g=0
$$

and has a solution

$$
g=\varphi_{1}(t ; x)=F(x,-x ; 1 ; t)=1-\frac{x^{2}}{(1 !)^{2}} t+\frac{x^{2}\left(x^{2}-1\right)}{(2 !)^{2}} t^{2}+\cdots,
$$

which is one of the hypergeometric functions (see [1]).

The function $F(x,-x ; 1 ; t)$ is the generating function for the polylogarithms

$$
\begin{aligned}
\operatorname{Li}_{a_{k}, \ldots, a_{1}}(t) & =\sum_{0<n_{1}<\cdots<n_{k}} \frac{t^{n_{k}}}{n_{1}^{a_{1}} \cdots n_{k}^{a_{k}}} \\
& =\int_{0<t_{a_{1}+\cdots+a_{k}}<\cdots<t_{1}<t} \frac{d t_{1}}{A_{\varepsilon_{1}}\left(t_{1}\right)} \cdots \frac{d t_{a_{1}+\cdots+a_{k}}}{A_{\varepsilon_{a_{1}+\cdots+a_{k}}}\left(t_{a_{1}+\cdots+a_{k}}\right)}
\end{aligned}
$$

(see [8] and (2.3)-(2.4) below). The latter integral is the Drinfeld-Kontsevich integral ([9]) with $\varepsilon_{j}=0$ or $\varepsilon_{j}=1, A_{0}(t)=t, A_{1}(t)=1-t$ and the sequence $\left(\varepsilon_{1}, \ldots, \varepsilon_{a_{1}-1}, \varepsilon_{a_{1}}, \varepsilon_{a_{1}+1}, \ldots, \varepsilon_{a_{1}+\cdots+a_{k}-1}, \varepsilon_{a_{1}+\cdots+a_{k}}\right)=(0, \ldots, 0,1,0, \ldots, 0,1)$, i.e. with 1 's in the places $a_{1}, a_{1}+a_{2}, \ldots, a_{1}+\cdots+a_{k}$.

The generating function $f_{2}(x)$ was computed in (1.3) due to the known product expansion of the function sin. The aim of the present paper is to calculate $f_{2}(x)$ directly from the hypergeometric equation using so-called WKB asymptotic expansions and Stokes operators.

The space of solutions to (1.6) is two-dimensional. Near $t=0$ the basic solutions are $\varphi_{1}(t ; x)$ (from (1.7)) and $\varphi_{2}(t ; x)=\varphi_{1}(t ; x) \ln \left(x^{2} t\right)+\varphi_{3}(t ; x)$, with an analytic germ $\varphi_{3}$. Near $s=1-t=0$ two independent solutions can be chosen in the form $\theta_{1}(s ; x)=x^{2} s+a_{2} s^{2}+\cdots$ and $\theta_{2}(s ; x)=$ $\theta_{1}(s ; x) \ln \left(x^{2} s\right)+b_{0}+b_{1} s+\cdots=\theta_{1}(s ; x) \ln \left(x^{2} s\right)+\theta_{3}(s ; x)$, where $b_{0}=-1$ and the other coefficients can be effectively calculated (see the next section). Therefore near $t=1$ the function $\varphi_{1}$ can be written as follows:

$$
\varphi_{1}(t ; x)=A(x) \cdot \theta_{1}(1-t ; x)+B(x) \cdot \theta_{2}(1-t ; x),
$$


and we have

$$
f_{2}(x)=\varphi_{1}(1 ; x)=B(x) b_{0}=-B(x) .
$$

So the problem is to find the connection coefficient $B(x)$. Here the solution $\varphi_{1}$ is defined uniquely, while to $\theta_{1}$ we can add const $\cdot \theta_{1}$, but the formula is invariant with respect to such changes.

The WKB method allows one to find another pair of solutions to the hypergeometric equation (1.6). These are formal asymptotic solutions as $x \rightarrow \infty$ of the following form:

$$
g_{0}^{ \pm}(t ; x) \sim e^{( \pm i x) S(t)}\left\{\frac{\psi_{1 / 2}(t)}{( \pm i x)^{1 / 2}}-\frac{\psi_{3 / 2}(t)}{( \pm i x)^{3 / 2}}+\cdots\right\} .
$$

Here the "action" $S(t)$ is $\int_{0}^{t}(\tau(1-\tau))^{-1 / 2} d \tau$ and the coefficient functions $\psi_{1 / 2}, \psi_{3 / 2}, \ldots$ satisfy a series of linear "transport equations"; we solve this system in Section 4.

The initial exponent $\gamma=-1 / 2$ of $x$ in (1.11) is calculated from the behavior of the solutions $\varphi_{1,2}(t ; x)$ as $x \rightarrow \infty, t \rightarrow 0$ but $y=x^{2} t$ is finite. We find $\varphi_{1,2}(t ; x) \approx \Phi_{1,2}(y)$, where $G=\Phi_{1,2}$ are basic solutions to the Bessel type equation

$$
\partial_{y} y \partial_{y} G+G=0
$$

in particular, we have $\Phi_{1}(y)=J_{0}(2 \sqrt{y})$ (where $J_{\mu}$ is the Bessel function, see (3.2) below). Next, one can use the asymptotic behavior of $J_{0}(r)$ as $r \rightarrow \infty$. Analogously we can look at the asymptotic behavior of the functions $\theta_{j}$ as $x \rightarrow \infty$ and $s=1-t \rightarrow 0$ and $z=x^{2} s$ is finite. It turns out that $\theta_{1,2}(s ; x) \approx \Theta_{1,2}(z)$, where $\Theta_{1,2}$ are basic solutions to another Bessel type equation

$$
z \partial_{z}^{2} H+H=0
$$

we have $\Theta_{1}(z)=\sqrt{z} J_{1}(2 \sqrt{z})$.

The idea is to expand the solution $\varphi_{1}$ in the WKB basis $\left(g_{0}^{+}, g_{0}^{-}\right)$, next represent the functions $g_{0}^{ \pm}$in the basis $\left(\theta_{1}, \theta_{2}\right)$ and find the desired representation of $\varphi_{1}$ in the basis $\left(\theta_{1}, \theta_{2}\right)$. Unfortunately, there is a problem with the series representing WKB solutions of differential equations with large parameter: they are generally divergent. They are also divergent in our case and the divergence is measured in terms of so-called Stokes matrices. To be more precise, there exist asymptotic series $g^{ \pm}(t ; x)$ analogous to $(1.11)$ which represent analytic functions in two sector-like domains in the $(t, x)$ variables (see Proposition 4.1 below). The relations between the WKB solutions $g^{ \pm}$ and the solutions (1.11) are of the form

$$
g^{ \pm}=C_{ \pm}\left(x^{-1}\right) g_{0}^{ \pm},
$$

where $C_{ \pm}\left(x^{-1}\right)=1+O\left(x^{-1}\right)$ are some asymptotic series. We shall show 
that $C_{+}\left(x^{-1}\right)=C_{-}\left(x^{-1}\right)=C\left(x^{-2}\right)$ and that

$$
\varphi_{1}=(1 / \sqrt{\pi})\left(g^{+}+g^{-}\right) ;
$$

in the proof we use some oscillatory integrals (see Lemma 4.4) or a direct reduction of the corresponding two-dimensional system to a normal diagonal form (see Remark 4.1). Therefore the solutions $g^{ \pm}$are more important that the solutions $g_{0}^{ \pm}$.

In general, it is hard to compare the solutions in these two domains, but it is possible to do it near $t=0$ (and $x \approx \infty$ ) and near $s=0$ (and $x \approx \infty$ ). In our next paper [16] we prove a theorem (see also Proposition 3.1 below) which says that the hypergeometric equation (1.6) is equivalent to the Bessel-like equation (1.12) via a transformation $\mathcal{H}_{0}$ which is analytic in $\left(t, x^{-1}\right) \in\left(\mathbb{C}^{2},(0,0)\right)$ (this is a generalization of a theorem from Wasow's book [13] about an analogous reduction to the Airy equation). For (1.12) the point $y=\infty$ is an irregular singular point and one finds there asymptotic solutions (also called WKB solutions) of the form

$$
G^{ \pm}(y)=e^{ \pm 2 i \sqrt{y}} y^{-1 / 4}\{1+\cdots\},
$$

also defined by divergent series but being analytic functions in larger sectors (than in the hypergeometric case). Anyway, it is possible to compare these WKB solutions in the intersections of adjacent sectors and in this way the Stokes operators arise.

The above mentioned theorem from [16] gives an equivalence of equation (1.6) rewritten using the "time" $s=1-t$ with the Bessel-like equation (1.12) and the equivalence matrix $\mathcal{H}_{1}$ is analytic in $s$ and $x^{-1}$. The basic WKB solutions to (1.6) are of the form

$$
H^{ \pm}(z)=e^{\mp 2 i \sqrt{z}} z^{1 / 4}\{1+\cdots\} .
$$

In the case of the hypergeometric equation (1.6) there exists an additional fundamental relation

$$
\theta_{1,2}(s ; x)=-s \partial_{s} \varphi_{1,2}(s ; x)
$$

between solutions near $t=0$ and near $t=1$ (see Proposition 2.1). One way to find the connection coefficient $B(x)$ uses the latter relation (and some calculus).

But there exists another way, which seems to admit a generalization to other equations (1.5) from Theorem 1.1. Namely analysis of the Stokes phenomena near the points $t=0$ and $t=1$ demonstrates that the connection coefficient $B(x)$ is represented by WKB type functions $x^{-1} e^{i \pi x}(1+\cdots)$ and $x^{-1} e^{-i \pi x}(1+\cdots)$ with trivial Stokes operators. So, the corresponding series are convergent and these WKB functions are single valued holomorphic functions on $\mathbb{C}$ near $x=\infty$ (see Theorem 5.1). Then it is easy to see that $B(x) /(\sin \pi x / x)$ is an entire and bounded function, hence a constant. 
Anyway, formulas obtained in this paper are new and seem beautiful. Moreover, we develop an approach to the Stokes phenomenon in the WKB method which has not been used before.

In the paper [17] in preparation we calculate some Stokes operators associated with a hypergeometric equation

$$
(1-t) \partial t \partial t \partial g+x^{3} g=0
$$

related to the generating function $f_{3}(x)$ associated with $\zeta(3)$ (compare Theorem 1.1). It turns out that $f_{3}(x)$ can be expressed by WKB type functions $x^{-3 / 2} e^{2 \pi \sigma x / \sqrt{3}}(1+\cdots), \sigma=-1, e^{i \pi / 3}, e^{-i \pi / 3}$. Moreover, it looks as if these WKB type summands are subject to a nontrivial Stokes phenomenon. In some sense the present paper is a testing work; it is easier to make calculations for $f_{2}(x)$ and check some conjectures which could be generalized to more general cases.

The plan of the paper is the following. In the next section we present the basic solutions of (1.6). In Section 3 we give some integral formulas for the Bessel functions related to the hypergeometric equation and their asymptotic behavior (via the stationary phase formula). In Section 4 we explore the WKB method for (1.6) with large $x$. In Section 5 we study the Stokes phenomenon associated with the WKB solutions.

\section{Solutions near $t=0$ and near $t=1$}

2.1. Solutions near $t=0$. The indicial equation (for solutions $g \sim t^{\alpha}$ ) associated with (1.6) near the singular point $t=0$ is $\alpha^{2}=0$. So we look for a solution of the form

$$
g=\varphi_{1}(t ; x)=1+a_{1} t+a_{2} t^{2}+\cdots
$$

and we get the recurrent relations $(n+1)^{2} a_{n+1}+\left(x^{2}-n^{2}\right) a_{n}=0$. The solution to this system gives the hypergeometric series $\varphi_{1}=F(x,-x ; 1, t)$ defined in (1.7). Next we look for another solution of the form

$$
g=\tilde{\varphi}_{2}(t ; x)=\varphi_{1} \cdot \ln t+b_{1} t+\cdots=\varphi_{1} \cdot \ln t+\varphi_{3}(t ; x) ;
$$

thus $\varphi_{3}$ satisfies the equation $\left\{(1-t) \partial t \partial+x^{2}\right\} \varphi_{3}+2(1-t) \partial \varphi_{1}=0$ and it is easy to see that the corresponding recurrent relations for $b_{n}$ are solvable. In particular, we have $b_{1}=-2 a_{1}=2 x^{2}$.

We can look at these solutions as functions of $x$. We write

$$
\begin{aligned}
& \varphi_{1}=\varphi_{1,0}(t)-\varphi_{1,1}(t) x^{2}+\varphi_{1,2}(t) x^{4}-\cdots, \\
& \tilde{\varphi}_{2}=\varphi_{2,0}(t)-\varphi_{2,1}(t) x^{2}+\varphi_{2,2}(t) x^{4}-\cdots,
\end{aligned}
$$

where $\varphi_{j, k}$ satisfy the system

$$
(t \partial)^{2} \varphi_{j, 0}=0, \quad(1-t) \partial t \partial \varphi_{j, k+1}=\varphi_{j, k} .
$$


The first equation has solutions $\varphi_{1,0}(t)=1$ and $\varphi_{2,0}(t)=\ln t$, and the other equations are solved by

$$
\varphi_{j, k+1}(t)=\int_{0}^{t} \frac{d t_{1}}{t_{1}} \int_{0}^{t_{1}} \frac{\varphi_{j, k}\left(t_{2}\right)}{1-t_{2}} d t_{2} .
$$

We get

$$
\varphi_{1,1}(t)=\sum_{n} \int_{0}^{t} \frac{d t_{1}}{t_{1}} \int_{0}^{t_{1}} t_{2}^{n} d t_{2}=\operatorname{Li}_{2}(t)
$$

(see (1.8)) and, generally,

$$
\varphi_{1, k}(t)=\mathrm{Li}_{2, \ldots, 2}(t)
$$

(with $k 2$ 's). Using the formula

$$
\int_{0}^{t} t_{1}^{n} \ln t_{1} d t_{1}=\frac{t^{n+1}}{n+1}\left(\ln t-\frac{1}{n+1}\right)
$$

we get $\varphi_{2,1}(t)=\mathrm{Li}_{2}(t) \ln t-2 \mathrm{Li}_{3}(t)$ and, generally,

$$
\varphi_{2, k}(t)=\mathrm{Li}_{2, \ldots, 2}(t) \cdot \ln t-2 \sum_{j=1}^{k} \operatorname{Li}_{2, \ldots, 3, \ldots, 2}(t),
$$

where the indices of the $\mathrm{Li}_{a_{1}, \ldots, a_{k}}$ are either $a_{1}=\cdots=a_{k}=2$, or $a_{j}=3$ and other $a_{i}=2$ (in the sum).

Remark 2.1. Putting $t=1$ in (2.4) and (2.5) we get $\varphi_{1, k}(1)=\zeta(2, \ldots, 2)$ (as expected) and $\varphi_{2, k}(1)=-2 \cdot \sum_{j=1}^{k} \zeta(2, \ldots, 3, \ldots, 2)$. After a simple resummation of the series we find

$$
\tilde{\varphi}_{2}(1)=2 x^{2} \cdot f_{2}(x) \cdot\left\{\zeta(3)+\zeta(5) x^{2}+\zeta(7) x^{4}+\cdots\right\} .
$$

Therefore the value at $t=1$ of the second solution to the hypergeometric equation defines a generating function for the zeta values at odd integers.

However we should not regard (2.6) as something important. The fact is that only the solution $\varphi_{1}$ is unique, while $\tilde{\varphi}_{2}$ can be changed to $\tilde{\varphi}_{2}+P(x) \varphi_{1}$ without affecting the basic properties of this solution, e.g. monodromy about $t=0$. Below we shall see that some other choices of the second (non-analytic) solution are more natural.

One of the choices of the second solution is

$$
\varphi_{2}(t ; x)=\varphi_{1}(t ; x) \cdot \ln \left(x^{2} t\right)+\varphi_{3}(t ; x) .
$$

2.2. Solutions near $t=1$. With the variable $s=1-t$ equation (1.6) takes the form

$$
s \partial_{s}(1-s) \partial_{s} g+x^{2} g=0
$$


where $\partial_{s}=\partial / \partial_{s}$. We easily find that the independent basic solutions can be chosen in the following form:

$$
\begin{aligned}
& \theta_{1}(s ; x)=x^{2} s-\frac{x^{2}\left(x^{2}-1\right)}{2 ! \cdot 1 !} s^{2}+\frac{x^{2}\left(x^{2}-1\right)\left(x^{2}-2^{2}\right)}{3 ! \cdot 2 !} s^{3}-\cdots, \\
& \tilde{\theta}_{2}(s ; x)=\theta_{1}(s ; x) \cdot \ln s-1+b_{1} s+\cdots=\theta_{1} \cdot \ln s+\theta_{3}(s ; x),
\end{aligned}
$$

where the function $\theta_{3}(s ; x)$ is analytic near $s=0$.

We shall also consider the following "second solution"

$$
\theta_{2}(s ; x)=\theta_{1} \cdot \ln \left(x^{2} s\right)+\theta_{3} .
$$

The following result is mentioned in the Introduction.

LEMMA 2.1. Let

$$
\varphi_{1}(t ; x)=A(x) \cdot \theta_{1}(1-t ; x)+B(x) \cdot \theta_{2}(1-t ; x)
$$

be the representation of the function $\varphi_{1}(t ; x)$ near $t=1$ in the basis $\theta_{1}, \theta_{2}$. Then the generating function $f_{2}$ for the zeta values equals

$$
f_{2}(x)=-B(x) .
$$

Finally, we note the following fundamental relation between solutions to the hypergeometric equation (1.6) near $t=0$ and near $s=1-t=0$.

Proposition 2.1. A function $g(t)$ is a solution to (1.6) if and only if the function

$$
h(s)=s \partial_{s} g(s)
$$

is a solution to (2.8). In particular,

$$
\theta_{1}(s ; x)=-s \partial_{s} \varphi_{1}(s ; x), \quad \theta_{2}(s ; x)=-s \partial_{s} \varphi_{2}(s ; x),
$$

where $\varphi_{1,2}$ are defined in Subsection 2.1. Moreover,

$$
\theta_{2}(s ; x)=-\varphi_{1}(0 ; x)+O(s), \quad s \rightarrow 0 .
$$

Proof. Putting $h(s)$ into (2.8) we get

$$
s \partial_{s}(1-s) \partial_{s} h+x^{2} h=s \partial_{s}\left[(1-s) \partial_{s} s \partial_{s} g+x^{2} g\right]=0 .
$$

Next, since the functions $s \partial_{s} \varphi_{1}(s)$ and $s \partial_{s} \varphi_{2}(s)$ are independent, they form a basis of solutions to $(2.8)$.

REMARK 2.2. The functions $\varphi_{1}(t ; x)$ and $\tilde{\varphi}_{2}(t ; x)$ are entire functions of $x$ (being the generating functions for polylogarithms). Moreover, for any fixed $x$ they are independent as functions of $t$. The same is true of $\theta_{1}(s ; x)$ and $\tilde{\theta}_{2}(s ; x)$.

Moreover, for $x=0$ near $t=1$ we have $\varphi_{1}(t ; 0)=1$ and $\tilde{\varphi}_{2}(t ; 0)=\ln t=$ $-s+O\left(s^{2}\right)$. This implies that the connection matrix $M(x)$ between the bases $\left(\varphi_{1}, \tilde{\varphi}_{2}\right)$ and $\left(\theta_{1}, \tilde{\theta}_{2}\right)$ is entire as a function of $x \in \mathbb{C}$. 


\section{Bessel type approximations and their asymptotic expansions}

3.1. Bessel approximations. Let us consider the series in (1.7) when $x \rightarrow \infty$. Then the recurrent relations for the coefficients in (2.1) become $a_{n+1} \approx-\left(\frac{x}{n+1}\right)^{2} a_{n}$ and we find that when $x \rightarrow \infty$ but $y=x^{2} t$ remains finite,

$$
\varphi_{1}(t ; x) \approx \Phi_{1}(y):=\sum_{n=0}^{\infty} \frac{(-y)^{n}}{(n !)^{2}}=J_{0}(2 \sqrt{y}) .
$$

Here and below,

$$
J_{\mu}(w)=\sum_{n=0}^{\infty} \frac{(-1)^{n}}{\Gamma(\mu+n+1) n !}\left(\frac{w}{2}\right)^{2 n+\mu}
$$

is the Bessel function with index $\mu$ (see [2, 6]).

The function $G=\Phi_{1}(y)$ satisfies the equation $\partial_{y} y \partial_{y} G+G=0$, obtained from (1.6) by replacing $t$ with $y / x^{2}$ and passing with $x$ to infinity; it is the Bessel type equation (1.12) from the Introduction.

Another solution to (1.12) is of the form

$$
\Phi_{2}(y)=\Phi_{1}(y) \ln y+\Phi_{3}(y)
$$

where $\Phi_{3}(y)=O(y)$ is an entire function of $y \in \mathbb{C}$.

We call a linear meromorphic equation of the Bessel type if it has only two singular points, at $t=0$ and at $t=\infty$ (see also [16]).

For solutions near $t=1$ we analogously find that when $x \rightarrow \infty$ but $z=x^{2} s$ is bounded,

$$
\theta_{1}(s ; x) \approx \Theta_{1}(z):=\sqrt{z} J_{1}(2 \sqrt{z}),
$$

where the function $\Theta_{1}(z)$ satisfies the Bessel type equation (1.13), with another solution

$$
\Theta_{2}(z)=\Theta_{1}(z) \ln z+\Theta_{3}(z)
$$

(where $\Theta_{3}(z)$ is an entire function).

The importance of the above approximations can be seen from the following result, which is a special case of a more general theorem proved in [16, Theorem 2].

Proposition 3.1. There exist matrix-valued functions $\mathcal{H}_{0}\left(t, x^{-1}\right)$ and $\mathcal{H}_{1}\left(s, x^{-1}\right)$, defined in a neighborhood of $(0,0) \in \mathbb{C}^{2}$ and analytic there, such that

$$
\left(\varphi_{1}, \varphi_{2}\right) \mathcal{H}_{0}=\left(\Phi_{1}, \Phi_{2}\right), \quad\left(\theta_{1}, \theta_{2}\right) \mathcal{H}_{1}=\left(\Theta_{1}, \Theta_{2}\right) .
$$

REMARK 3.1. Let

$$
\mathcal{F}_{0}=\left(\begin{array}{cc}
\varphi_{1} & \varphi_{2} \\
\partial \varphi_{1} & \partial \varphi_{2}
\end{array}\right), \quad \mathcal{G}_{0}=\left(\begin{array}{cc}
\Phi_{1} & \Phi_{2} \\
\partial \Phi_{1} & \partial \Phi_{2}
\end{array}\right)
$$


be the fundamental matrices associated with the bases $\left(\varphi_{1}, \varphi_{2}\right)$ (see $(2.1)$ and (2.7)), and $\left(\Phi_{1}, \Phi_{2}\right)$ (see (3.1) and (3.3)), and $\partial \Phi_{j}=x^{2} \partial_{y} \Phi_{j}$ means differentiation with respect to the time $t$. Then we have

$$
\mathcal{H}_{0}(t ; x)=\mathcal{F}_{0}^{-1} \mathcal{G}_{0} .
$$

Analogously the fundamental matrices

$$
\mathcal{F}_{1}=\left(\begin{array}{cc}
\theta_{1} & \theta_{2} \\
\partial_{s} \theta & \partial_{s} \theta_{2}
\end{array}\right), \quad \mathcal{G}_{1}=\left(\begin{array}{cc}
\Theta_{1} & \Theta_{2} \\
\partial_{s} \Theta_{1} & \partial_{s} \Theta_{2}
\end{array}\right)
$$

(where $\partial_{s}=x^{2} \partial_{z}$ ) define the matrix-valued function

$$
\mathcal{H}_{1}(s ; x)=\mathcal{F}_{1}^{-1} \mathcal{G}_{1} .
$$

It was proved in [16] that the matrices $\mathcal{H}_{0,1}$ are analytic in $\left(t, x^{-1}\right)$ and in $\left(s, x^{-1}\right)$ (respectively) near $(0,0)$. (In the proof one uses the fact that the matrices $\mathcal{F}_{0}$ and $\mathcal{G}_{0}$ have the same monodromy properties as $t$ goes around 0 and as $x$ goes around $\infty$ and have almost the same asymptotics as $t \rightarrow 0$ and $x \rightarrow \infty$, e.g. in sectorial domains.)

Theorem 2 from [16] is a generalization of a theorem of $\mathrm{W}$. Wasow from [13] about reduction of equations of the form $\ddot{x}=\lambda^{2} t a(t)+\lambda b(t, 1 / \lambda)$, $a(0)=1$ (with analytic germs $a$ and $b$ and large $\lambda$ ) to the Airy equation $\partial_{T}^{2} y=T y, T=t \lambda^{2 / 3}$, which is also of the Bessel type.

3.2. Integral formulas. Bessel functions admit representations via contour integrals.

LEMma 3.1. The following formula holds for all integer $n$ :

$$
J_{n}(2 \sqrt{y})=\frac{1}{2 \pi} \int_{-\pi}^{\pi} \exp (2 i \sqrt{y} \sin \alpha) e^{-i n \alpha} d \alpha .
$$

Proof. Formula (3.6) was obtained by Bessel and can be found in the literature (see [2, 6] ). Let us recall the simple argument that can be used in more general situations.

We have

$$
\begin{aligned}
J_{n}(2 \sqrt{y}) & =\sum_{m=0}^{\infty} \frac{(-1)^{m} y^{m+n / 2}}{(m+n) ! m !} \\
& =\operatorname{res}_{u=0} \frac{1}{u^{n+1}}\left(\sum \frac{(\sqrt{y} u)^{m}}{m !}\right)\left(\sum \frac{(-\sqrt{y} / u)^{m}}{m !}\right) \\
& =\frac{1}{2 \pi i} \int_{|u|=1} \exp (\sqrt{y}(u-1 / u)) \frac{d u}{u^{n+1}} .
\end{aligned}
$$

Putting $u=e^{i \alpha}$ we get the result. 
Lemma 3.2. For a non-integer index $\mu$ we have the following Schläfli representation:

$$
\begin{aligned}
J_{\mu}(w)= & \frac{1}{2 \pi} \int_{-\pi}^{\pi} \exp (i(w \sin \alpha-\mu \alpha)) d \alpha \\
& -\frac{\sin \pi \mu}{\pi} \int_{0}^{\infty} \exp (-w \sinh \beta-\mu \beta) d \beta .
\end{aligned}
$$

Proof. This follows from some generalization of the residue formula for $J_{n}$ with integer $n$. We have

$$
J_{\mu}(w)=\frac{1}{2 \pi i} \int_{C} \exp \left(\frac{1}{2} w(u-1 / u)\right) u^{-\mu-1} d u
$$

where $C$ is a contour which begins and ends at $u=-\infty$ and surrounds $u=0$ in the positive direction. Next the contour $C$ is deformed to two half-lines along $(-\infty,-1)$ (parametrized by $\left.-e^{\beta}\right)$ and the circle $|u|=1$. For more details we refer the reader to [2, formula 7.3(9)] (see also the proof of Lemma 4.2 below).

In the original Schläfli formula the first integral in (3.7) is replaced with $\pi^{-1} \int_{0}^{\pi} \cos (w \sin \alpha-\mu \alpha) d \alpha$.

Lemma 3.3. The solution $\Phi_{2}$ to the Bessel type equation (1.12) can be defined as

$$
\Phi_{2}(y)=\lim _{\nu \rightarrow 0} \frac{1}{\nu}\left\{J_{\nu}(2 \sqrt{y})-J_{-\nu}(2 \sqrt{y})\right\}-2 \gamma \Phi_{1}(y),
$$

where $\gamma$ is the Euler-Mascheroni constant. It admits the following integral formula:

$$
\begin{aligned}
\Phi_{2}(y)+2 \gamma \Phi_{1}(y)= & \frac{1}{i \pi} \int_{-\pi}^{\pi} \alpha \exp (2 i \sqrt{y} \sin \alpha) d \alpha \\
& -2 \int_{0}^{\infty} \exp (-2 \sqrt{y} \sinh \beta) d \beta
\end{aligned}
$$

where the function $\Phi_{2}(y)$ is given in formula (3.3).

Proof. Take the following perturbation of (1.12):

$$
\left\{\left(y \partial_{y}\right)^{2}+y-\nu^{2} / 4\right\} G=0,
$$

where $\nu \neq 0$ is a small parameter. Its independent solutions are $J_{\nu}(2 \sqrt{y})$ and $J_{-\nu}(2 \sqrt{y})$, as can be easily checked. Of course, the right hand side of (3.8) defines a solution to the unperturbed equation. Next, we have 


$$
\begin{aligned}
J_{\nu}(2 \sqrt{y}) & =\frac{1}{\Gamma(1+\nu)} y^{\nu / 2}\left\{1-\frac{y}{(1+\nu) 1 !}+\frac{y^{2}}{(1+\nu)(2+\nu) 2 !}-\cdots\right\} \\
& =J_{0}(2 \sqrt{y})\{1-\nu \Psi(1)+(\nu / 2) \ln y\}+\nu\left\{c_{1} y+c_{2} y^{2}+\cdots\right\}+O\left(\nu^{2}\right)
\end{aligned}
$$

(see $(3.2))$, where

$$
\Psi(z)=\Gamma^{\prime}(z) / \Gamma(z)
$$

is the Euler Psi-function (with $\Psi(1)=-\gamma$ ).

To prove formula (3.9) we first note that $\lim _{\nu \rightarrow 0} \nu^{-1}\left\{J_{\nu}(2 \sqrt{y})-J_{-\nu}(2 \sqrt{y})\right\}$ $=\left.2 \frac{\partial}{\partial \nu} J_{\nu}(2 \sqrt{y})\right|_{\nu=0}$. Next we use the Schläfli formula (3.7).

3.3. Oscillatory integrals. Recall (see [7]) that the stationary phase formula concerns integrals of the type

$$
I(\lambda)=\int e^{\lambda \phi(\alpha)} \chi(\alpha) d^{k} \alpha
$$

over a $k$-dimensional manifold when $|\lambda| \rightarrow \infty$. Assuming that the "phase" $\phi(\alpha)$ has finitely many critical points $\alpha_{1}, \ldots, \alpha_{n}$, which are Morsean, one has the following asymptotic stationary phase formula:

$$
I(\lambda) \sim \sum_{i} \chi\left(\alpha_{i}\right) \frac{1}{\sqrt{\operatorname{det}\left(-D^{2} \phi\left(\alpha_{i}\right)\right)}} e^{\lambda \phi\left(\alpha_{i}\right)}\left(\frac{2 \pi}{\lambda}\right)^{k / 2} .
$$

Usually, in applications, the large parameter $\lambda$ is imaginary and the phase $\phi$ is a real function; then the integral in (3.10) is called an oscillatory integral. This is the case considered in this paper.

3.4. Asymptotics for $\Phi_{j}$ and $\Theta_{j}$. In the integral (3.10), specified to (3.6), the phase function $\phi(\alpha)=2 i \sin \alpha$ has two critical points $\alpha_{1}=\pi / 2$ with $\phi\left(\alpha_{1}\right)=2 i, \phi^{\prime \prime}\left(\alpha_{1}\right)=-2 i$ and $\alpha_{2}=-\pi / 2$ with $\phi\left(\alpha_{2}\right)=-2 i, \phi^{\prime \prime}\left(\alpha_{2}\right)$ $=2 i$. Therefore we obtain the following (well known) asymptotic formula for $y \rightarrow \infty$ :

$$
\Phi_{1}(y)=J_{0}(2 \sqrt{y}) \sim \frac{1}{2 \sqrt{\pi} y^{1 / 4}}\left(e^{i(2 \sqrt{y}-\pi / 4)}+e^{-i(2 \sqrt{y}-\pi / 4)}\right) .
$$

On the right hand side of (3.9) the second integral can be ignored, because it decreases like $y^{-1 / 2}$ (without any exponent). The first integral in that formula is an oscillatory integral and a standard application of (3.11) gives $($ for $y \rightarrow \infty$ )

$$
\Phi_{2}(y)+2 \gamma \Phi_{1}(y) \sim \frac{\sqrt{\pi}}{2 i y^{1 / 4}}\left(e^{i(2 \sqrt{y}-\pi / 4)}-e^{-i(2 \sqrt{y}-\pi / 4)}\right) .
$$

Concerning the solutions $\Theta_{j}(z)$ to the Bessel type equation (1.13), we could find integral formulas (analogous to those above). But it is more natural to use Proposition 2.1, which implies

$$
\Theta_{j}(z)=-z \partial_{z} \Phi_{j}(z) .
$$


Therefore (3.12)-(3.13) give (for $z \rightarrow \infty$ )

$$
\begin{aligned}
\Theta_{1}(z) & \sim \frac{z^{1 / 4}}{2 i \sqrt{\pi}}\left(e^{i(2 \sqrt{z}-\pi / 4)}-e^{-i(2 \sqrt{z}-\pi / 4)}\right), \\
\Theta_{2}(z)+2 \gamma \Theta_{1}(z) & \sim-\frac{\sqrt{\pi}}{2} z^{1 / 4}\left(e^{i(2 \sqrt{z}-\pi / 4)}+e^{-i(2 \sqrt{z}-\pi / 4)}\right) .
\end{aligned}
$$

REMARK 3.2. The above asymptotic formulas are true when $y$ is real (respectively when $z$ is real). In the case of a non-real argument only one term, which dominates, is correct. This is related to the Stokes phenomenon discussed in Section 5.

4. The WKB solutions. Equation (1.5) for a large parameter $x$ theoretically can be solved using the WKB method. This means that one represents a solution as a finite sum of terms of the form

$$
e^{x S(t)}\left\{\psi_{\gamma}(t) x^{-\gamma}+\psi_{\gamma+1}(t) x^{-\gamma-1}+\cdots\right\} .
$$

In general the series in the above formula are divergent, but this divergence can be somehow controlled. Below we present three approaches to the WKB solutions to (1.6): formal, via the stationary phase formula and via normal forms.

The name of the method comes from the names of its authors, G. Wentzel [14, H. Kramers [10] and L. Brillouin [4]. Originally it was used to solve approximately the Schrödinger equation [11], but here we apply it to the hypergeometric equation.

4.1. Formal WKB solutions to the hypergeometric equation. We look for so-called WKB solutions to the hypergeometric equation (1.6) of the form $g=e^{x \tilde{S}(t)} \psi(t ; x)$. Substituting this to (1.6) we get the equation

$$
\begin{aligned}
\left\{(1-t) t(\partial \tilde{S})^{2}+1\right\}+x^{-1}(1-t)\{2(t \partial \tilde{S}) \partial \psi+(\partial(t \partial \tilde{S})) \psi\} & \\
+ & x^{-2}(1-t) \partial(t \partial \psi)=0 .
\end{aligned}
$$

This implies the "Hamilton-Jacobi equation"

$$
t(1-t)(\partial \tilde{S})^{2}=-1
$$

with two solutions

$$
\tilde{S}(t)= \pm i \int_{0}^{t} \frac{d \tau}{\sqrt{\tau(1-\tau)}}=: \pm i S(t)
$$

Thus we get two independent solutions to the hypergeometric equation, $g_{0}^{ \pm}(t ; x)=e^{ \pm i x S(t)} \psi^{ \pm}(t ; x)$.

Let

$$
x_{ \pm}= \pm i x
$$


Then we can take the following asymptotic expansion (valid for both solutions):

$$
g_{0}^{ \pm}(t ; x)=e^{x_{ \pm} S(t)}\left\{\psi_{1 / 2}(t) x_{ \pm}^{-1 / 2}-\psi_{3 / 2}(t) x_{ \pm}^{-3 / 2}+\psi_{5 / 2}(t) x_{ \pm}^{-5 / 2}+\cdots\right\},
$$

where

$$
x_{ \pm}^{1 / 2}=e^{ \pm i \pi / 4} \sqrt{x} \quad(\text { for } x>0)
$$

and the functions $\psi_{j}$ satisfy the "transport equations"

$$
\begin{aligned}
2(t \partial S) \partial \psi_{1 / 2}+\left(\partial\left(t \partial S_{2}\right)\right) \psi_{1 / 2} & =0, \\
2(t \partial S) \partial \psi_{j}+\left(\partial\left(t \partial S_{2}\right)\right) \psi_{j} & =\partial\left(t \partial \psi_{j-1}\right), \quad j>1 / 2 .
\end{aligned}
$$

We choose a solution to (4.5) in the form

$$
\psi_{1 / 2}(t)=(t \partial S)^{-1 / 2}=\sqrt[4]{\frac{1-t}{t}} .
$$

To solve the other equations we introduce the new variable

$$
u=\sqrt[4]{\frac{t}{1-t}}
$$

thus

$$
t=\frac{u^{4}}{1+u^{4}}, \quad \partial=\partial_{t}=\frac{\left(1+u^{4}\right)^{2}}{4 u^{3}} \partial_{u}
$$

and

$$
\psi_{1 / 2}(t)=\tilde{\psi}_{1 / 2}(u)=1 / u \text {. }
$$

The functions $\psi_{j}(t)=\tilde{\psi}_{j}(u), j>1 / 2$, satisfy the equations

$$
\tilde{\psi}_{j}^{\prime}+\frac{1}{u} \tilde{\psi}_{j}=\frac{1}{8 u^{2}}\left(u\left(1+u^{4}\right) \tilde{\psi}_{j-1}^{\prime}\right)^{\prime},
$$

where the prime denotes $\partial_{u}$. The recursive solutions to (4.10) have the form

$$
\tilde{\psi}_{j}=T \tilde{\psi}_{j-1}, \quad(T \varphi)(u)=\frac{1}{8 u} \int^{u} \frac{1}{w}\left(w\left(1+w^{4}\right) \varphi^{\prime}(w)\right)^{\prime} d w .
$$

We find

$$
\tilde{\psi}_{3 / 2}=\frac{-1}{2 \cdot 8}\left(u^{-3}+3 u\right), \quad \tilde{\psi}_{5 / 2}=\frac{3}{8^{3}}\left(3 u^{-5}-5 u^{3}\right)
$$

and $\tilde{\psi}_{7 / 2}=-\frac{3 \cdot 5}{2 \cdot 8^{4}}\left(5 u^{-7}+7 u^{5}\right)-\frac{9 \cdot 5}{2 \cdot 8^{4}}\left(u^{-3}+3 u\right), \tilde{\psi}_{9 / 2}=\frac{3 \cdot 5^{2} \cdot 7}{2 \cdot 8^{6}}\left(7 u^{-9}-9 u^{7}\right)+$ $\frac{3 \cdot 5 \cdot 11}{2 \cdot 8^{5}}\left(3 u^{-5}-5 u^{3}\right)$.

These formulas suggest introduction of the functions

$$
\omega_{n}(u)=(n-2) u^{-n}+(-1)^{n(n+1) / 2} n \cdot u^{n-2}, \quad n=3,5,7, \ldots
$$


One easily verifies the following recurrent relations:

$$
\begin{aligned}
T \omega_{3} & =-\frac{3 \cdot 1}{8 \cdot 4} \omega_{5}, \\
T \omega_{n} & =-\frac{n(n-2)}{8(n+1)} \omega_{n+2}-\frac{n(n-2)}{8(n-3)} \omega_{n-2}, \quad n=5,7, \ldots
\end{aligned}
$$

It follows that

$$
\begin{aligned}
\psi_{n / 2}(t) & =\tilde{\psi}_{n / 2}(u) \\
& =a_{n, n} \omega_{n}(u)+a_{n, n-4} \omega_{n-4}(u)+\cdots, \quad n=3,5, \ldots,
\end{aligned}
$$

for some coefficients $a_{n, m}$ which are calculated inductively and grow very fast with $n$; for instance, $a_{n, n}=(n-2)(-1 / 8)^{(n-3) / 2}((n-4) ! !)^{2} /(n-3) ! !$.

The solution (4.11) to (4.10) is only a particular solution; a general solution would contain the term const $\cdot u^{-1}$. But the Laurent polynomials $\tilde{\psi}_{n / 2}(u)$, $n=3,5, \ldots$, in $(4.13)$ do not contain the term with $u^{-1}$ proportional to $\tilde{\psi}_{1 / 2}$.

Recall that the hypergeometric equation is related to some Bessel type equations. Therefore these Bessel type equations should also admit solutions of WKB type. Indeed, the Bessel type equations (1.12) and (1.13) have $y=\infty$ (respectively $z=\infty$ ) as an irregular singular point (see [19]). As suggested by the asymptotic formulas (3.12)-(3.15) they admit formal asymptotic solutions

$$
G^{ \pm}(y)=e^{ \pm 2 i \sqrt{y}} y^{-1 / 4}\left\{1-\frac{a_{1}}{ \pm i y^{1 / 2}}+\frac{a_{2}}{\left( \pm i y^{1 / 2}\right)^{2}}-\frac{a_{3}}{\left( \pm i y^{1 / 2}\right)^{3}}+\cdots\right\}
$$

and

$$
H^{ \pm}(z)=e^{ \pm 2 i \sqrt{z}} z^{1 / 4}\left\{1-\frac{b_{1}}{ \pm i z^{1 / 2}}+\frac{b_{2}}{\left( \pm i z^{1 / 2}\right)}-\frac{b_{3}}{\left( \pm i z^{1 / 2}\right)^{3}}+\cdots\right\},
$$

where the coefficients $a_{j}$ and $b_{j}$ are defined recursively (compare (1.15)$(1.16))$.

Definition 4.1. We define the testing WKB solutions $g_{0}^{ \pm}(t ; x)$ by the right hand side of (4.3) where $\psi_{j}$ are defined by (4.9) and (4.11) with the condition that $\psi_{j}, j>1 / 2$, do not contain the monomial $u^{-1}$. In other words, $\psi_{j}$ are defined as in (4.13).

The (formal) function $G^{ \pm}(y)$ and $H^{ \pm}(z)$ are called the WKB solutions for the Bessel type equations (1.12) and (1.13) respectively.

\subsection{Integral representations and a stationary phase formula.} The solutions to the hypergeometric equation admit integral formulas given in the lemmas below. 
Lemma 4.1. We have

$$
\varphi_{1}=F(x,-x ; 1 ; t)=\frac{1}{2 \pi i} \oint_{|v|=1}\left(\frac{1+\sqrt{t} v}{1+\sqrt{t} / v}\right)^{x} \frac{d v}{v} .
$$

Proof. This follows from the residue formula and the expansions

$$
\begin{gathered}
(1+\sqrt{t} v)^{x}=1-x(-\sqrt{t} v)+\frac{x(x-1)}{2 !}(-\sqrt{t} v)^{2}+\cdots, \\
(1+\sqrt{t} / v)^{-x}=1+x(-\sqrt{t} / v)+\frac{x(x+1)}{2 !}(-\sqrt{t} / v)^{2}+\cdots .
\end{gathered}
$$

(This representation is slightly different from the standard Euler integral.)

The next lemma is a generalization of the Schläfli formula.

Lemma 4.2. For $\operatorname{Re}(\gamma-\beta)>0$ and $0<t<1$ we have

$$
\begin{aligned}
& \frac{\Gamma(\gamma-\beta)}{\Gamma(1-\beta) \Gamma(\gamma)} \cdot t^{(\gamma-1) / 2} \cdot F(\alpha, \beta ; \gamma ; t) \\
& =\frac{1}{2 \pi} \int_{-\pi}^{\pi}\left(1+\sqrt{t} e^{i \varphi}\right)^{\gamma-\beta-1}\left(1+\sqrt{t} e^{-i \varphi}\right)^{-\alpha} e^{i(1-\gamma) \varphi} d \varphi \\
& \quad+\frac{\sin \pi(\gamma+\alpha)}{\pi} \int_{1}^{1 / \sqrt{t}}(1-\sqrt{t} w)^{\gamma-\beta-1}(1-\sqrt{t} / w)^{-\alpha} \frac{d w}{w^{\gamma}}
\end{aligned}
$$

Proof. We have the following analogue of the Euler integral:

$$
\begin{aligned}
& \frac{\Gamma(\gamma-\beta) t^{(\gamma-1) / 2}}{\Gamma(1-\beta) \Gamma(\gamma)} F(\alpha, \beta ; \gamma ; t) \\
& \quad=\frac{1}{2 \pi i} \int_{\gamma}(1+\sqrt{t} v)^{\gamma-\beta-1}(1+\sqrt{t} / v)^{-\alpha} \frac{d v}{v^{\gamma}},
\end{aligned}
$$

where $\Upsilon$ is a contour in the complex $v$-plane which begins and ends at $v=$ $-1 / \sqrt{t}$ and surrounds the point $v=0$ in the counterclockwise direction.

To prove (4.18) we expand the factor $(1+\sqrt{t} / v)^{-\alpha}=\sum \frac{\Gamma(\alpha+n)}{\Gamma(\alpha) n !} t^{n / 2}(-v)^{-n}$ and then we use the Euler Beta function relation

$$
\begin{aligned}
(-1)^{n} \int_{\Upsilon}(1+\sqrt{t} v)^{\gamma-\beta-1} v^{-n-\gamma} d v & \\
= & \left(e^{i \pi \gamma}-e^{-i \pi \gamma}\right) \int_{-1 / \sqrt{t}}^{0}(1+\sqrt{t} v)^{\gamma-\beta-1}(-v)^{-n-\gamma} d v
\end{aligned}
$$




$$
\begin{aligned}
& =2 i \sin \pi \gamma \cdot t^{(n+\gamma-1) / 2} \frac{\Gamma(\gamma-\beta) \Gamma(-n-\gamma+1)}{\Gamma(-n-\beta+1)} \\
& =2 i \sin \pi \gamma \cdot t^{(n+\gamma-1) / 2} \cdot \Gamma(\gamma-\beta) \cdot \frac{\Gamma(\beta+n) \sin \pi \beta}{\Gamma(\gamma+n) \sin \pi \gamma},
\end{aligned}
$$

where $\sin \pi \beta=\pi / \Gamma(1-\beta) \Gamma(\beta)$.

Finally one deforms the contour $\Upsilon$ to the unit circle $\left\{v=e^{i \varphi}:-\pi<\varphi\right.$ $<\pi\}$ and two straight line segments along $\{v=-w: 1 \leq w \leq 1 / \sqrt{t}\}$.

Lemma 4.3. The second basic solution near $t=0$ to the hypergeometric equation (1.6) (for $\operatorname{Re} x>0$ ) can be taken in the form

$$
\begin{aligned}
\hat{\varphi}_{2} & =\frac{1}{2 \pi i} \int_{|v|=1}\left(\frac{1+\sqrt{t} v}{1+\sqrt{t} / v}\right)^{x} \ln \left(\frac{1+\sqrt{t} v}{v^{2}(1+\sqrt{t} / v)}\right) \frac{d v}{v} \\
& -\int_{1}^{1 / \sqrt{t}}\left(\frac{1-\sqrt{t} v}{1-\sqrt{t} / v}\right)^{x}\left\{\frac{\sin \pi x}{\pi} \ln \left(\frac{1-\sqrt{t} v}{v^{2}(1-\sqrt{t} / v)}\right)+3 \cos \pi x\right\} \frac{d v}{v} .
\end{aligned}
$$

Proof. We take the family of Riemann equations $t\left\{(1-t) \partial t \partial g+x^{2} g\right\}-$ $\mu^{2} g=0, \mu \approx 0$; it is a perturbation of (1.6). It has the solutions $\eta_{\mu}(t ; x)$ and $\eta_{-\mu}(t ; x)$, where

$$
\begin{aligned}
\eta_{\mu}= & \frac{\Gamma(1+x+\mu)}{\Gamma(1+x-\mu) \Gamma(1+2 \mu)} \cdot t^{\mu} \cdot F(\mu+x, \mu-x ; 1+2 \mu ; t) \\
= & \varphi_{1}+\mu\{2 \Psi(1+x)-2 \Psi(1)+\ln t\} \cdot \varphi_{1} \\
& +\left.\mu \frac{\partial}{\partial \mu}\right|_{\mu=0} F(\mu+x, \mu-x ; 1+2 \mu ; t)+O\left(\mu^{2}\right)
\end{aligned}
$$

and $\Psi$ denotes the Euler Psi-function. It follows that $\hat{\varphi}_{2}=\lim _{\mu \rightarrow 0} \frac{1}{2 \mu}\left\{\eta_{\mu}-\right.$ $\left.\eta_{-\mu}\right\}$ is a solution to (1.6) with the logarithmic term. Now, using the generalized Schläfli formula (4.17) with $\alpha=\mu+x, \beta=\mu-x$ and $\gamma=1+2 \mu$, we get

$$
\eta_{\mu}=\frac{1}{2 \pi i} \int\left(\frac{1+\sqrt{t} v}{1+\sqrt{t} / v}\right)^{x+\mu} \frac{d v}{v^{1+2 \mu}}-\frac{\sin \pi(x+3 \mu)}{\pi} \int\left(\frac{1-\sqrt{t} v}{1-\sqrt{t} / v}\right)^{x+\mu} \frac{d v}{v^{1+2 \mu}},
$$

where the path of integration in the first integral is $\left\{v=e^{i \alpha}: 0<\alpha<2 \pi\right\}$ and the corresponding path in the second integral is the interval $[1,1 / \sqrt{t}]$.

Remark 4.1. Since

$$
F(\mu+x, \mu-x ; 1+2 \mu ; t)=1+\frac{\mu^{2}-x^{2}}{1+2 \mu} t+\cdots,
$$

it follows that the analytic part $\varphi_{3}(t)=O(t)$ of the solution $\tilde{\varphi}_{2}=\varphi_{1} \ln t+\varphi_{3}$ 
from (2.2) equals

$$
\varphi_{3}(t)=\left.\frac{\partial}{\partial \mu} F(\mu+x, \mu-x ; 1+2 \mu ; t)\right|_{\mu=0}=2 x^{2} t+O\left(t^{2}\right) .
$$

Moreover, from the expansion $\Psi(1+x)=-\gamma+\zeta(2) x-\zeta(3) x^{2}+\zeta(4) x^{3}-\cdots$ (see [1, Eq. 1.17(5)]) and (2.6) in Remark 2.1 we get

$$
\hat{\varphi}_{2}=\tilde{\varphi}_{2}+(2 \Psi(1+x)-2 \Psi(1)) \cdot \varphi_{1}
$$

and

$$
\hat{\varphi}_{2}(1 ; x)=f_{2}(x)\left\{2 \zeta(2) x+2 \zeta(4) x^{3}+2 \zeta(6) x^{5}+\cdots\right\} .
$$

But $\frac{\pi}{\tan \pi x}=\frac{1}{x}-2 \zeta(2) x-2 \zeta(4) x^{3}-\cdots$ (compare [1, Eq. 1.21(3)]). Therefore $\hat{\varphi}_{2}(1 ; x)=-\frac{\cos \pi x}{x}+x^{-1} f_{2}(x)$. This implies that the function

$$
\check{\varphi}_{2}(t ; x):=\hat{\varphi}_{2}(t ; x)-x^{-1} \cdot \varphi_{1}(t ; x)
$$

is a solution to (1.6) independent of $\varphi_{1}$ and such that

$$
\check{\varphi}_{2}(1 ; x)=-\frac{\cos \pi x}{x} .
$$

There is also a natural relation between the solution $\hat{\varphi}_{2}$ and the solution $\varphi_{2}=\tilde{\varphi}_{2}+\varphi_{1} \ln x^{2}$ (see (2.7)). Indeed, from the Stirling formula it follows that $\Psi(1+x) \sim \ln x+\frac{1}{2 x}+O\left(x^{-2}\right)$ as $x \rightarrow \infty$. Therefore, as $x \rightarrow \infty$, we have

$$
\hat{\varphi}_{2} \simeq \varphi_{2}+\left(2 \gamma+O\left(x^{-1}\right)\right) \varphi_{1}
$$

(compare (3.9) above).

Anyway, we have found four candidates for the second solution to (1.6): $\tilde{\varphi}_{2}, \varphi_{2}, \hat{\varphi}_{2}$ and $\check{\varphi}_{2}$. All are natural and reasonable.

Having integral formulas for solutions to our equation (1.6) we can use the stationary phase formula. We begin with (4.16).

The integral in (4.16), with $v=e^{i \alpha}$ and large $x>0$, is an oscillatory integral (see (3.10)) with the "phase"

$$
\phi=\frac{1}{i}\{\ln (1+\sqrt{t} v)-\ln (1+\sqrt{t} / v)\} .
$$

The stationary phase formula states that the leading contribution to the integral in (4.16) comes from neighborhoods of critical points of the phase. There are two critical points $\alpha_{ \pm}$which correspond to $v_{ \pm}=e^{i \alpha_{ \pm}}=-\sqrt{t} \mp$ $i \sqrt{1-t}$. The corresponding critical values of the phase are

$$
\phi_{ \pm}=\mp \frac{1}{i} \ln \frac{1-i u^{2}}{1+i u^{2}}= \pm S(t), \quad u=\sqrt[4]{\frac{t}{1-t}},
$$

where $S(t)=\int_{0}^{t}(\tau(1-\tau))^{-1 / 2} d \tau$ is the same as in (4.2) above.

The critical points $\alpha_{ \pm}$are non-degenerate. We can see this by putting $v=v_{ \pm} e^{i a}$ into (4.24) and expanding $\phi$ in powers of (small) $a$. We find 
$\phi(\alpha)=\phi_{ \pm} \mp u^{2} a^{2}+\cdots$, or $i x \phi=x_{ \pm} S(t)-x_{ \pm} u^{2} a^{2}+\cdots, x_{ \pm}= \pm i x$. Therefore the leading term of the oscillatory integral corresponding to the critical point $\alpha_{ \pm}$equals

$$
e^{x_{ \pm} S(t)} \cdot \frac{1}{2 \pi} \int \exp \left(-x_{ \pm} u^{2} a^{2}\right) d a \sim \frac{1}{2 u \sqrt{\pi x_{ \pm}}} e^{x_{ \pm} S(t)} .
$$

Let us now look at the further terms of the integral in (4.16). For this we put $a=A /\left(u \sqrt{x_{ \pm}}\right)$and expand

$$
i x \Delta_{ \pm} \phi:=i x\left(\phi-\phi_{ \pm}\right)
$$

in powers of $x_{ \pm}^{-1 / 2}$. We get

$$
\begin{aligned}
i x \Delta_{ \pm} \phi= & \pm i x_{ \pm} \ln \left(1 \mp i u^{2}\left(e^{i A / u \sqrt{x_{ \pm}}}-1\right)\right) \\
& \mp i x_{ \pm} \ln \left(1 \mp i u^{2}\left(e^{-i A / u \sqrt{x_{ \pm}}}-1\right)\right) .
\end{aligned}
$$

The $x_{ \pm}^{0}$-term of this expression equals $-A^{2}$, and the other terms, denoted by $\Omega(A)$, can be grouped as follows:

$$
\begin{aligned}
x_{ \pm} u^{2}\left[\sum_{m \geq 0, n \geq 2} c_{m, n} u^{4 m}\left(\frac{A^{2}}{u^{2} x_{ \pm}}\right)^{n}\right] & \\
& +\left( \pm i \sqrt{x_{ \pm}} u^{3} A\right)\left[\sum_{m \geq 0, n \geq 1} d_{m, n} u^{4 m}\left(\frac{A^{2}}{u^{2} x_{ \pm}}\right)^{n}\right]
\end{aligned}
$$

for some real coefficients $c_{m, n}$ and $d_{m, n}$ (which do not depend on the sign \pm ). We get an integral of the form

$$
\frac{1}{2 \pi u \sqrt{x_{ \pm}}} \int e^{-A^{2}} \times e^{\Omega} d A
$$

where $e^{\Omega(A)}$ is expanded in powers of $A$ and integrated. By analogy with the Gaussian integrals we can assume that

$$
\left\langle A^{n}\right\rangle:=\frac{1}{\sqrt{\pi}} \int e^{-A^{2}} A^{n} d A=(n-1) ! ! \cdot\left(\frac{1}{2}\right)^{n / 2}
$$

if $n$ is even, and zero otherwise.

We have computed a few initial terms in the expansion of $\varphi_{1}(t ; x)$ and we have found that it is a sum of two asymptotic series $\frac{1}{2}\left(\pi x_{ \pm}\right)^{-1 / 2} e^{x_{ \pm} S(t)}\left\{\frac{1}{u}+\right.$ $\left.\frac{1}{16} x_{ \pm}^{-1}\left(u^{-3}+3 u\right)-2 \cdot 8^{-3} x_{ \pm}^{-2}\left(\frac{10}{u}-3\left(3 u^{-5}-5 u^{3}\right)\right)+\cdots\right\}$, i.e. $\frac{1}{2} \pi^{-1 / 2} e^{x \pm S(t)}$ times

$$
\left\{\tilde{\psi}_{1 / 2}(u) x_{ \pm}^{-1 / 2}-\tilde{\psi}_{3 / 2}(u) x_{ \pm}^{-3 / 2}+\left(\frac{5}{256} \tilde{\psi}_{1 / 2}(u)+\tilde{\psi}_{5 / 2}(u)\right) x_{ \pm}^{-5 / 2}+\cdots\right\}
$$

(compare (4.12)); we do not present these rather tedious calculations here. Anyway, we can state the following result. 
LEMMA 4.4. We have

$$
\varphi_{1} \sim \frac{1}{2 \sqrt{\pi}} C\left(x^{-2}\right)\left(g_{0}^{+}+g_{0}^{-}\right)=: \frac{1}{2 \sqrt{\pi}}\left(g^{+}+g^{-}\right),
$$

where $C\left(x^{-2}\right)$ is a formal series with real coefficients such that

$$
C\left(x^{-2}\right)=1+\frac{5}{256 x^{2}}+\cdots \not \equiv 1 .
$$

Proof. We prove only the formula (4.27). The expansion of $e^{\Omega}$, with $\Omega$ given in (4.26), gives a sum of the following monomials in $u$ and $x_{ \pm}$:

$$
\begin{array}{r}
u^{4 \sum k_{j} m_{j}+4 \sum k_{j}^{\prime} m_{j}^{\prime}-2 \sum k_{j}\left(n_{j}-1\right)-\sum k_{j}^{\prime}\left(2 n_{j}^{\prime}-3\right)} \cdot x_{ \pm}^{-\sum k_{j}\left(n_{j}-1\right)-\sum k_{j}^{\prime}\left(n_{j}^{\prime}-1 / 2\right)} \\
=u^{4 M+4 M^{\prime}-2 N+2 K-2 N^{\prime}+3 K^{\prime}} \cdot x_{ \pm}^{-N+K-N^{\prime}+K^{\prime} / 2}
\end{array}
$$

where $M=\sum k_{j} m_{j}, M^{\prime}=\sum k_{j}^{\prime} m_{j}^{\prime}, N=\sum k_{j} n_{j}, N^{\prime}=\sum k_{j}^{\prime} n_{j}^{\prime}, K=\sum k_{j}$, $K^{\prime}=\sum k_{j}^{\prime}$ (and the primes are related to the expansion of the exponent of the second sum in (4.26)). We are interested in the monomials involving $u^{0}$.

Since there are no terms with half-integer powers of $x_{ \pm}\left(\operatorname{as}\left\langle A^{2 n+1}\right\rangle=0\right)$, we see that $K^{\prime}=2 K^{\prime \prime}$ is an even integer. Therefore the condition $4 M+4 M^{\prime}-$ $2 N-2 N^{\prime}+2 K+6 K^{\prime \prime}=0$ implies $N+N^{\prime}-K-K^{\prime \prime}=2\left(M+M^{\prime}+K^{\prime \prime}\right)$, i.e. an even power of $x_{ \pm}$.

DEFINITION 4.2. The formal functions

$$
g^{\sigma}=C\left(x^{-2}\right) \cdot g_{0}^{\sigma}
$$

are called the principal WKB solutions to (1.6).

Consider now the solution $\hat{\varphi}_{2}$ defined by (4.19). The right hand side of (4.19) consists of two integrals, of which only the first, along $\left\{v=e^{i \alpha}: 0<\right.$ $\alpha<2 \pi\}$, is essential when applying the stationary phase formula.

We have an oscillatory integral $I=\int e^{i x \phi(\alpha)} \chi(\alpha) d \alpha$ with the same phase as in (4.21) but with different amplitude $\chi(\alpha)=\frac{i}{2 \pi}(\phi(\alpha)-2 \alpha)$. We write

$$
\hat{\varphi}_{2} \sim I=I_{+}+I_{-},
$$

where $I_{ \pm}$are contributions from neighborhoods of the critical points $\alpha_{ \pm}$of the phase. Since

$$
\chi\left(\alpha_{ \pm}+a\right)=\frac{\mp i}{2}+\frac{i}{2 \pi} \Delta_{ \pm}(a)-\frac{i}{\pi} a,
$$

we get

$$
I_{ \pm}=J_{ \pm}+K_{ \pm}+L_{ \pm}
$$

where the integrals $J_{ \pm}, K_{ \pm}, L_{ \pm}$correspond to the three summands in $\chi$. 
LEMMA 4.5. We have

$$
\hat{\varphi}_{2} \sim \frac{\sqrt{\pi}}{2 i}\left\{D_{+}\left(x^{-1}\right) g_{0}^{+}-D_{-}\left(x^{-1}\right) g_{0}^{-}\right\},
$$

where $D_{ \pm}\left(x^{-1}\right)$ are formal series satisfying

$$
D_{+}\left(x^{-1}\right)+D_{-}\left(x^{-1}\right)=2 C\left(x^{-2}\right) .
$$

Proof. Of course, $J_{ \pm}= \pm i \pi \cdot C\left(x^{-2}\right) g_{0}^{ \pm}$.

We shall prove that the integrals $K_{+}+K_{-}$and $L_{+}+L_{-}$(from (4.32)) are both proportional to $g_{0}^{+}+g_{0}^{-}$; this is sufficient. For this it is enough to show that the Gauss type integrals $\int e^{i x \Delta_{ \pm}} \Delta_{ \pm}\left(\right.$in $\left.K_{ \pm}\right)$and $\frac{1}{u} x_{ \pm}^{-1 / 2} \int A e^{-A^{2}} e^{\Omega} d A$ (in $L_{ \pm}$) have "correct" coefficients of $u^{-1}$.

Consider first the integral related to $L_{ \pm}$. Repeating the expansion of $e^{\Omega}$ from the proof of Lemma 4.4 we find monomials of the form

$$
\pm i \cdot u^{4 M+4 M^{\prime}-2 N+2 K-2 N^{\prime}+3 K^{\prime}-1} \cdot x_{ \pm}^{-N+K-N^{\prime}+K^{\prime} / 2-1 / 2}
$$

where $K^{\prime}=2 K^{\prime \prime}+1$ must be odd. The condition $4 M+4 M^{\prime}-2 N+2 K-2 N^{\prime}+$ $6 K^{\prime \prime}+2=0$ implies that the latter monomial equals $\pm i \cdot x_{ \pm}^{2\left(M+M^{\prime}+K^{\prime \prime}\right)+1}=$ $(-1)^{M+M^{\prime}+K^{\prime \prime}+1} \cdot x^{2\left(M+M^{\prime}+K^{\prime \prime}\right)+1}$, i.e. it does not depend on the sign \pm .

Note that

$$
\begin{aligned}
e^{-x_{ \pm} S} K_{ \pm} & =\frac{i}{2 \pi} \int e^{i x \Delta_{ \pm} \phi} \Delta_{ \pm} \phi d a=\frac{\partial}{\partial x} \frac{1}{2 \pi} \int e^{i x \Delta_{ \pm} \phi} d a \\
& =\frac{1}{2 \sqrt{\pi}} \frac{\partial}{\partial x} C\left(x^{-2}\right)\left\{u^{-1} x_{ \pm}^{-1 / 2}+\cdots\right\}
\end{aligned}
$$

(compare above). The coefficient of $u^{-1} x_{ \pm}^{-1 / 2}$ on the right hand side equals $(1 / 2 \sqrt{\pi})\left\{-2 x^{-3} C^{\prime}\left(x^{-2}\right)-\frac{1}{2} C\left(x^{-2}\right) x^{-1}\right\} \stackrel{1}{=}-1 / 4 \sqrt{\pi} x$ and does not depend on the sign \pm .

More precise calculations show that $K_{+}+K_{-}+L_{+}+L_{-}=(1 / 2 x \sqrt{\pi}$ $+\cdots)\left\{g_{0}^{+}+g_{0}^{-}\right\}$.

4.3. Reduction to a normal form. Putting $h=\dot{g} / x$ we rewrite (1.6) as the first order system

$$
\left(\begin{array}{l}
\dot{g} \\
\dot{h}
\end{array}\right)=A(t ; x)\left(\begin{array}{l}
g \\
h
\end{array}\right)
$$

where

$$
A=x A_{1}(t)+A_{0}(t), \quad A_{1}=\left(\begin{array}{cc}
0 & 1 \\
1 / t(t-1) & 0
\end{array}\right), \quad A_{0}=\left(\begin{array}{cc}
0 & 0 \\
0 & -1 / t
\end{array}\right) .
$$

The normal form of such a system is a diagonal (or independent) system obtained by means of a formal linear change which depends on $t$. 
The first step is the diagonalization of the matrix $A_{1}(t)$ with the eigenvalues $\pm \lambda(t)$,

$$
\lambda(t)=i / \sqrt{t(1-t)} .
$$

We put

$$
X=\lambda(t) g+h, \quad Y=-\lambda(t) g+h
$$

and we get

$$
\begin{aligned}
& \dot{X}=\lambda(t) x X-\frac{1}{4}\left(\frac{3}{t}-\frac{1}{1-t}\right) X-\frac{1}{4}\left(\frac{1}{t}+\frac{1}{1-t}\right) Y, \\
& \dot{Y}=-\lambda(t) x Y-\frac{1}{4}\left(\frac{1}{t}+\frac{1}{1-t}\right) X-\frac{1}{4}\left(\frac{3}{t}-\frac{1}{1-t}\right) Y .
\end{aligned}
$$

The general theory says that such a system can be diagonalized by means of an infinite series of "shearing" transformations (see [5, 7]). Let us apply some initial transformations, in order to compare the (partial) normal form obtained with the results of the previous and next subsections. We put

$$
\begin{aligned}
& X=X_{1}+\left(\frac{b_{1}(t)}{x}+\frac{b_{2}(t)}{x^{2}}+\cdots\right) Y_{1}, \\
& Y=\left(\frac{c_{1}(t)}{x}+\frac{c_{2}(t)}{x^{2}}+\cdots\right) X_{1}+Y_{1},
\end{aligned}
$$

and we expect to obtain the following separated system:

$$
\begin{aligned}
& \dot{X}_{1}=\left(\lambda(t) x+\lambda_{0}(t)+\frac{\lambda_{1}(t)}{x}+\cdots\right) X_{1}, \\
& \dot{Y}_{1}=\left(-\lambda(t) x+\mu_{0}(t)+\frac{\mu_{1}(t)}{x}+\cdots\right) Y_{1} .
\end{aligned}
$$

The resulting system of equations in $b_{j}, c_{j}, \lambda_{j}$ and $\mu_{j}$ is easily solved. Using the variable $u=(t /(1-t))^{1 / 4}$ (see (4.8)) we get

$$
\begin{aligned}
& b_{1}=-c_{1}=\frac{-i}{8(t(1-t))^{1 / 2}}=-i \frac{1+u^{4}}{8 u^{2}}, \\
& b_{2}=c_{2}=\frac{1-2 t}{32 t(1-t)}=\frac{1-u^{8}}{32 u^{4}}
\end{aligned}
$$

and

$$
\begin{aligned}
& \lambda_{0}=\mu_{0}=-\frac{1}{4}\left(\frac{3}{t}-\frac{1}{1-t}\right), \\
& \lambda_{1}=-\mu_{1}=-\frac{i}{32(t(1-t))^{3 / 2}}=\frac{-i}{32} \frac{\left(1+u^{4}\right)^{3}}{u^{6}}, \\
& \lambda_{2}=\mu_{2}=\frac{-1}{128} \frac{1-2 t}{t^{2}(1-t)^{2}}=\frac{\left(u^{4}-1\right)\left(1+u^{4}\right)^{4}}{128 u^{8}} .
\end{aligned}
$$


General solutions to the system (4.39) are of the form

$$
\begin{aligned}
X_{1}= & K_{1} \cdot \frac{e^{i x S(t)}}{t^{3 / 4}(1-t)^{1 / 4}} \\
& \times \exp \left\{\frac{-i}{16 x}\left(u^{2}-\frac{1}{u^{2}}\right)-\frac{1}{512 x^{2}}\left(u^{4}+2+\frac{1}{u^{4}}\right)+\cdots\right\}, \\
Y_{1}= & K_{2} \cdot \frac{e^{-i x S(t)}}{t^{3 / 4}(1-t)^{1 / 4}} \\
& \times \exp \left\{\frac{i}{16 x}\left(u^{2}-\frac{1}{u^{2}}\right)-\frac{1}{512 x^{2}}\left(u^{4}+2+\frac{1}{u^{4}}\right)+\cdots\right\},
\end{aligned}
$$

with arbitrary constants $K_{1,2}$ (which may depend on $x$ ). Substituting this to (4.36) and then to $g=\frac{1}{2 \lambda}(X-Y)$ (see (4.35)) one finds a general solution to (1.6) in the form

$$
g=K_{1} \tilde{g}^{+}(t ; x)+K_{2} \tilde{g}^{-}(t ; x),
$$

where

$$
\tilde{g}^{ \pm}(t ; x)=\left(1+\frac{5}{256 x^{2}}+\cdots\right) g_{0}^{ \pm}(t ; x)
$$

and $g_{0}^{ \pm}$are the testing WKB solutions (see Definition 4.1).

REMARK 4.2. The solutions $\tilde{g}^{ \pm}$are also WKB solutions. They seem to be more important than the testing WKB solutions $g_{0}^{ \pm}$, because they are represented by analytic functions in some sectorial domains (due to a theorem of Birkhoff discussed below).

In fact the relation between $\tilde{g}^{ \pm}$and $g_{0}^{ \pm}$is of the form

$$
\tilde{g}^{ \pm}(t ; x)=\tilde{C}\left(x^{-2}\right) \cdot g_{0}^{ \pm}(t ; x),
$$

where $\tilde{C}\left(x^{-2}\right)$ is a formal series. This can be seen by a closer look at the above reduction to the normal form, i.e. $b_{2 j+1}=-c_{2 j+1}, \lambda_{2 j+1}=-\mu_{2 j+1}$ and $b_{2 j}=c_{2 j}, \lambda_{2 j}=\mu_{2 j}$.

This and the coincidence of (4.42) with (4.28) above suggest that the functions $\tilde{g}^{ \pm}$coincide with the principal WKB solutions $g^{ \pm}$from Definition 4.2. We do not know how to prove this. Anyway, we have the relation $\tilde{g}^{ \pm}=(\tilde{C} / C) g^{ \pm}$.

Note also that the normal form system (4.39) is more natural than the WKB solutions $\tilde{g}^{ \pm}$, because the latter involve the initial condition $S(0)=0$.

The reduction (4.38) is divergent (as a power series in $x^{-1}$ ) and the WKB solutions $\tilde{g}^{ \pm}$are only formal solutions. G. Birkhoff [3] was the first to prove that such a system can be diagonalized analytically in some sectorial domains. Below we present a scheme of Birkhoff's proof. 
In the first step we apply a change of variables

$$
X=X_{1}+U(t) Y_{1}, \quad Y=V(t) X_{1}+Y_{1}
$$

which should transform the system (4.20), i.e.

$$
\left(\begin{array}{l}
\dot{X} \\
\dot{Y}
\end{array}\right)=\left(\begin{array}{ll}
A^{11} & A^{12} \\
A^{21} & A^{22}
\end{array}\right)\left(\begin{array}{l}
X \\
Y
\end{array}\right)
$$

to the diagonal form

$$
\dot{X}_{1}=B_{1}(t) X_{1}, \quad \dot{Y}_{1}=B_{2}(t) Y_{1} .
$$

We get $B_{1}=A^{11}-V A^{21}, B_{2}=A^{21} U+A^{22}$ and two independent Riccati equations

$$
\begin{aligned}
& \dot{U}=A^{11} U-U A^{22}+A^{12}-U A^{21} U, \\
& \dot{V}=A^{22} V-V A^{11}+A^{21}-V A^{12} V .
\end{aligned}
$$

The latter differential equations can be rewritten in the form of the integral equations

$$
\begin{aligned}
& U(t)=\int_{\Gamma_{1}(t)} e^{P(t)-P(\tau)}\left\{A^{12}(\tau)-U(\tau) A^{21}(\tau) U(\tau)\right\} d \tau, \\
& V(t)=\int_{\Gamma_{2}(t)} e^{P(\tau)-P(t)}\left\{A^{21}(\tau)-V(\tau) A^{12}(\tau) V(\tau)\right\} d \tau,
\end{aligned}
$$

where $P(t)=\int_{0}^{t}\left(A^{11}(\iota)-A^{22}(\iota)\right) d \iota=2 i x S(t)+\cdots$. Here $\Gamma_{1}(t)$ and $\Gamma_{2}(t)$ are suitable paths in the $\tau$-plane.

We shall consider two domains

$$
\begin{aligned}
& \mathcal{D}_{u}=\{\operatorname{Im} x S(t)>-\alpha, \operatorname{Im} x(\pi-S(t))>-\alpha,|x|>1 / \varepsilon, t \in \mathcal{W}\}, \\
& \mathcal{D}_{d}=\{\operatorname{Im} x S(t)<\alpha, \operatorname{Im} x(\pi-S(t))<\alpha,|x|>1 / \varepsilon, t \in \mathcal{W}\},
\end{aligned}
$$

where $\alpha>0$ is a fixed constant and

$$
\mathcal{W}=\left\{t=t_{1}+i t_{2}: \varepsilon<t_{1}<1-\varepsilon,\left|t_{2}\right|<\varepsilon t_{1}\left(1-t_{1}\right)\right\}
$$

is a small neighborhood of the segment $(\varepsilon, 1-\varepsilon) \subset \mathbb{R} \subset \mathbb{C}$ and $\varepsilon>0$ is a small constant.

If $(t, x) \in \mathcal{D}_{u}$ (from "up"), then the contour $\Gamma_{1}(t)$ begins at $\tau=\tau_{0}>0$ (where $\tau_{0} \ll \varepsilon$ ) and ends at $\tau=t$ and is such that $\operatorname{Im} x(S(t)-S(\tau))>0$ along $\Gamma_{1}(t)$, and the path $\Gamma_{2}(t)$ begins at $\tau=1-\tau_{0}$ and ends at $\tau=t$ and has $\operatorname{Im} x(S(t)-S(\tau))<0$. In this case (4.47)-(4.48) can be solved by using the contraction principle, because the factor $\exp (P(t)-P(\tau))$ in (4.4) is uniformly bounded (respectively the factor $\exp (P(\tau)-P(t))$ in (4.48) is uniformly bounded). Moreover, the solutions are analytic in the variables $t$ and $x$ in the domain $\mathcal{D}_{u}$ and satisfy $U \sim O(1 / x)$ and $V \sim O(1 / x)$. We refer the reader to [13, 19] for details. 
Solving the diagonal system (4.44), substituting the solution to (4.43) (with $U$ and $V$ just obtained) and then inserting $X$ and $Y$ into $g=$ $(X-Y) / 2 \lambda$ we get the following analogue of (4.41):

$$
g=K_{1} g_{u}^{+}(t ; x)+K_{2} g_{u}^{-}(t ; x), \quad(t, x) \in \mathcal{D}_{u} .
$$

For $(t, x) \in \mathcal{D}_{d}$ (from "down") the path $\Gamma_{1}(t)$ is the same as $\Gamma_{2}(t)$ defined above, and $\Gamma_{2}(t)$ becomes the old $\Gamma_{1}(t)$. Here also (4.47)-(4.48) have unique solutions which are analytic in $\mathcal{D}_{d}$. We get the following analogue of (4.50):

$$
g=K_{1} g_{d}^{+}(t ; x)+K_{2} g_{d}^{-}(t ; x), \quad(t, x) \in \mathcal{D}_{d} .
$$

We note the conjugation symmetry of the above construction:

$$
\overline{g_{u}^{+}(t ; x)}=g_{d}^{-}(\bar{t} ; \bar{x}), \quad \overline{g_{u}^{-}(t ; x)}=g_{d}^{+}(\bar{t} ; \bar{x}) .
$$

Of course, the solutions $g_{u, d}^{ \pm}$are defined modulo multiplication by functions of $x$, i.e. the constants $K_{1,2}$ above can be functions of $x$ (analytic in suitable domains). We normalize the solutions $g_{u, d}^{ \pm}$by the condition

$$
\varphi_{1}=\frac{1}{2 \sqrt{\pi}}\left(g_{u}^{+}+g_{u}^{-}\right)=\frac{1}{2 \sqrt{\pi}}\left(g_{d}^{+}+g_{d}^{-}\right), \quad x>0,0<t<1,
$$

in agreement with Lemma 4.4 above (see (4.27)) and with Definition 4.2.

Let us summarize the results of this subsection in the following

Proposition 4.1. There exist analytic WKB solutions $\left(g_{u}^{+}, g_{u}^{-}\right)$, analytic and continuous in $\mathcal{D}_{u}$, and $\left(g_{d}^{+}, g_{d}^{-}\right)$, analytic and continuous in $\mathcal{D}_{d}$, whose formal expansions are the same as for the formal WKB solutions $\left(g^{+}, g^{-}\right)$from Definition 4.2. They satisfy the relations (4.52) and (4.53).

REMARK 4.3. One can choose another way to diagonalize the system (4.37). One can first use the transformation $X=X_{1}+U(t) Y$, which leads to the triangular system $\dot{X}_{1}=B_{1} X_{1}, \dot{Y}=B_{21} X_{1}+B_{2} Y$. This leads to the same Riccati equation for $U$ as (4.45), which is solved in the same way. Then one applies the transformation $Y=Y_{1}+V(t) X_{1}$ to diagonalize the triangular system. But the equation for $V$ is simpler than (4.46), it is a linear equation with an explicit solution of the form $V(t)=\int_{\Gamma_{2}(t)} e^{Q(\tau)-Q(t)} B_{21}(\tau) d \tau$ (for a suitable function $Q$ ). But it turns out that the composition of the latter triangular changes of variable is different from (4.43). The difference is of the same type as in the Stokes phenomenon. Moreover, this approach spoils the conjugation symmetry (4.52).

The paths $\Gamma_{j}(t)$ do not begin at $\tau=0$ or at $\tau=1$ due to the poles of the functions $A^{i j}(t)$ at $t=0$ and at $t=1$. The different choices of $\tau_{0}$ also result in the Stokes phenomenon. Probably this can be avoided by some regularization of the divergent integrals in (4.47)-(4.48).

Recall that Riccati equations are related to second order linear differential equations. A natural question is which linear equation appears and how 
it relates to the original hypergeometric equation. But calculations show that the coefficients of the linear equation are multivalued, expressed via $\sqrt{t(1-t)}$, and we do not see anything interesting here.

REMARK 4.4. An analogue of analytic diagonalization in the domains $\mathcal{D}_{u}$ and $\mathcal{D}_{d}$ of the hypergeometric system (4.35) is the sectorial normalization theorem for systems related to the Bessel type equation (1.12).

First one applies the ramified change of the "time" variable $y=v^{2}$, which leads to the equation

$$
\partial_{v} v \partial_{v} \tilde{G}+4 v \tilde{G}=0
$$

(for $\tilde{G}=G \circ v^{2}$ ), or to the system

$$
\frac{d}{d v} G_{1}=G_{2}, \quad \frac{d}{d v} G_{2}=-4 G_{1}-\frac{1}{v} G_{2} .
$$

Using the variables $X=2 G_{1}-i G_{2}, Y=2 G_{1}+i G_{2}$ we get the system

$$
\frac{d X}{d v}=2 i X-\frac{1}{2 v}(X-Y), \quad \frac{d Y}{d v}=-2 i Y+\frac{1}{2 v}(X-Y)
$$

which should be diagonalized. The analytic diagonalization uses the transformation (4.44) with the Riccati equations (4.45)-(4.46), equivalent to the integral equations (4.47)-(4.48). However, in (4.47)-(4.48) the integration paths $\Gamma_{1}=\Gamma_{1}(v)$ and $\Gamma_{2}=\Gamma_{2}(v)$ are different. They end up at $\tau=v$, are parallel to the real axis and, depending on the sector considered, they begin either at $\tau=+\infty$ or at $\tau=-\infty$. The sectors about $v=\infty$ are

$$
\mathcal{S}_{r}=\{-\pi+\delta<\arg v<\pi-\delta\}, \quad \mathcal{S}_{l}=\{\delta<\arg v<2 \pi-\delta\}
$$

for a small constant $\delta>0$. Therefore there exist two reductions to the normal (diagonal) form, one analytic in the sector $\mathcal{S}_{r}$ ("right") and one analytic in the sector $\mathcal{S}_{l}$ ("left").

Analogously the Bessel type equation (1.13) rewritten as a system with new time $w=\sqrt{z}$, i.e.

$$
w \partial_{w} w^{-1} \partial_{w} \tilde{H}+4 \tilde{H}=0,
$$

is transformed to the diagonal form by means of two transformations which are analytic in the sectors (4.55) about $w=\infty$.

4.4. WKB solutions associated with $t=1$. The testing WKB solutions $g_{0}^{ \pm}$from Definition 4.1 have the exponential term $e^{ \pm i x S(t)}$ which equals 1 for $t=0$ (and $e^{ \pm i x \pi}$ for $t=1$ ). Therefore introduction of the following functions seems natural; they will be explored in the next section.

Definition 4.3. Together with the solutions $g_{0}^{ \pm}$(from Definition 4.1) and $g^{ \pm}$(from Definition 4.2) we have the following WKB solutions (associ- 
ated with the point $s=1-t=0)$ :

$$
\begin{aligned}
& h_{0}^{ \pm}(s ; x)=x_{ \pm} e^{-i x_{ \pm} S(1)} g_{0}^{ \pm}(1-s ; x), \\
& h^{ \pm}(s ; x)= \pm i x e^{\mp i x \pi} g^{ \pm}(1-s ; x) .
\end{aligned}
$$

By Proposition 2.1 and Lemmas 4.4 and 4.5 we have

$$
\theta_{1}(s ; x)=-\frac{C\left(x^{-2}\right)}{2 \sqrt{\pi}}\left\{s \partial_{s} g_{0}^{+}(s ; x)+s \partial_{s} g_{0}^{-}(s ; x)\right\}
$$

and a second solution can be taken in the form

$$
\begin{aligned}
\hat{\theta}_{2}(s ; x) & =-s \partial_{s} \hat{\varphi}_{2}(s ; x) \\
& \sim-\frac{\sqrt{\pi}}{2 i}\left\{D_{+}\left(x^{-1}\right) s \partial_{s} g_{0}^{+}(s ; x)-D_{-}\left(x^{-1}\right) s \partial_{s} g_{0}^{-}(s ; x)\right\} .
\end{aligned}
$$

Since $\hat{\varphi}_{2}=\varphi_{2}+$ const $\cdot \varphi_{1}$, also $\hat{\theta}_{2}=\theta_{2}+$ const $\cdot \theta_{1}$, and hence $(2.9)$ gives $\hat{\theta}_{2}(0 ; x)=\theta_{2}(0 ; x)=-1$.

We have

$$
s \partial_{s}\left[e^{x_{ \pm} S(s)} \psi^{ \pm}(s ; x)\right]=\left\{x_{ \pm} \sqrt{\frac{s}{1-s}} \psi^{ \pm}+s \partial_{s} \psi^{ \pm}\right\} e^{x_{ \pm} S} .
$$

Using $u=\sqrt[4]{t /(1-t)}=\sqrt[4]{(1-s) / s}$ and $s \partial_{s}=-\left(\left(1+u^{4}\right) / 4 u^{3}\right) \partial_{u}$, we find that $\psi^{ \pm}(s ; x)=u x_{ \pm}^{-1 / 2}+\cdots$ is expanded into a series of $\omega_{n}(1 / u)=$ $(n-2) u^{n}+(-1)^{n(n+1) / 2} n u^{2-n}$ with coefficients depending on $x$. We have $\left(\left(1+u^{4}\right) / 4 u^{3}\right) \partial_{u} u=\frac{1}{4}\left(u^{-3}+u\right)$ and

$$
\begin{aligned}
& \frac{1+u^{4}}{4 u^{3}} \partial_{u} \omega_{n}(1 / u) \\
& \quad=\frac{n(n-2)}{4} \frac{\left(1+u^{4}\right)\left((-1)^{n(n+1) / 2}+u^{2 n-2}\right)}{u^{3}}, \quad n=3,5, \ldots .
\end{aligned}
$$

We see that the first term (with $x^{1 / 2}$ ) in the expansion of the right hand side of (4.59) equals $x_{ \pm}^{1 / 2} / u$ (is proportional to $1 / u$ ) and the other terms (with $\left.x^{j}, j<1 / 2\right)$ in $x_{ \pm} \sqrt{s /(1-s)} \psi^{ \pm}$and in $s \partial_{s} \psi^{ \pm}$do not contain the monomial $u^{-1}$.

Next,

$$
S(s)=S(1)-S(t)=\pi-S_{2}(t) .
$$

This, together with the above, implies the following important identity:

$$
s \partial_{s} g_{0}^{ \pm}(s ; x)=x e^{ \pm i \pi x} g_{0}^{\mp}(t ; x)=\mp i h_{0}^{\mp}(s ; x), \quad t=1-s .
$$

This, together with the results of the previous subsection, yields 
LEMMA 4.6. We have

$$
\begin{aligned}
& \theta_{1}(s ; x) \sim-x \frac{C}{2 \sqrt{\pi}}\left\{e^{i \pi x} g_{0}^{-}(t ; x)+e^{-i \pi x} g_{0}^{+}(t ; x)\right\} \\
& \hat{\theta}_{2}(s ; x) \sim x \frac{\sqrt{\pi}}{2 i}\left\{-D_{+} e^{i \pi x} g_{0}^{-}(t ; x)+D_{-} e^{-i \pi x} g_{0}^{+}(t ; x)\right\} .
\end{aligned}
$$

This implies the formulas

$$
\begin{aligned}
& \hat{\varphi}_{1}(t ; x)=-\frac{D_{+} e^{i \pi x}+D_{-} e^{-i \pi x}}{x\left(D_{+}+D_{-}\right)} \theta_{1}(s ; x)-\frac{2 C}{D_{+}+D_{-}} \frac{\sin \pi x}{\pi x} \hat{\theta}_{2}(s ; x), \\
& \hat{\varphi}_{2}(t ; x)=\frac{\pi i}{x} \frac{D_{+}^{2} e^{i \pi x}-D_{-}^{2} e^{-i \pi x}}{C\left(D_{+}+D_{-}\right)} \theta_{1}(s ; x)+\frac{D_{+} e^{i \pi x}+D_{-} e^{-i \pi x}}{x\left(D_{+}+D_{-}\right)} \hat{\theta}_{2}(s ; x) .
\end{aligned}
$$

This lemma, the equalities $\theta_{1}(0 ; x)=0$ and $\hat{\theta}_{2}(0 ; x)=-1$ and $(4.34)$ (in Lemma 4.5) imply

Corollary 4.1. We have $f_{2}(x)=-B(x)=\sin \pi x / \pi x$.

REMARK 4.5. The above proof of the formula for the generating function $f_{2}(x)$, although looking natural, is not of the sort which could be generalized to other generating functions.

Moreover, it seems that the series $g_{0}^{ \pm}(t ; x), C\left(x^{-2}\right)$ and $D_{ \pm}\left(x^{-1}\right)$ do not correspond to concrete functions analytic in any domains. One should rather use functions $g^{ \pm}(t ; x)$ (instead of $\left.g_{0}^{ \pm}\right)$and $D_{ \pm} / C$ (instead of $\left.D_{ \pm}\right)$which correspond to functions analytic in the domains $\mathcal{D}_{u, d}$ (see Proposition 4.1). This point of view is assumed in the next section.

5. Stokes operators. Usually the Stokes phenomenon [12] is related to normalization of a linear system $\dot{z}=A(t) z$ in a neighborhood of an irregular singular point, say at $t=0$. The neighborhood of $t=0$ is divided into sectors $\mathcal{S}_{j}$ such that there exist transformations $z=\mathcal{B}_{j}(t) y$ holomorphic with respect to $t \in \mathcal{S}_{j}$ which lead to a diagonal system $\dot{y}=\operatorname{diag}\left(d_{1}(t), \ldots, d_{n}(t)\right) y$. But the matrix-valued functions $H_{j}$ are different in different sectors. The difference between $\mathcal{B}_{j}$ and $\mathcal{B}_{j+1}$ is measured via so-called Stokes matrices (see [19]).

In the context of WKB solutions, e.g. for $t \in(0,1)$ and $x$ large, usually the Stokes matrices are related to solutions near one of the endpoints of the time interval, $t=0$ or $t=1$ (see [7]). One would like to define analogues of the Stokes operators for the WKB solutions, but when the time $t \in(0,1)$ is real and $x$ varies in some sectors near $x=\infty$, i.e. in $(\mathbb{C}, \infty)$. However, a rather detailed analysis performed in [16] demonstrates that it is not possible to do this in a uniform way with respect to $t$. Moreover, calculations of the Stokes operators associated with the third order hypergeometric equation (1.18) demonstrate that the Stokes operators at the two endpoints of the interval $(0,1)$ are essentially different. 
When studying the Stokes phenomenon in [7] and [5] greater attention is focused on analytic properties of the WKB solutions with respect to the time $t$, while the parameter $x \approx+\infty$ is usually real. The so called Stokes lines are drawn in the complex $t$-plane near the "turning points" $t=0$ and $t=1$. In this section we focus our attention on the parameter $x$, which will vary in whole sectors near infinity, and the time $t$ will vary in a small neighborhood of the interval $(\varepsilon, 1-\varepsilon) \subset \mathbb{C}$ (as in Subsection 4.3).

5.1. Stokes operators for Bessel type equations. We begin with equation (1.12). It has (uniquely defined) formal WKB solutions $G^{+}(y) \sim$ $y^{-1 / 4} e^{2 i \sqrt{y}}$ and $G^{-}(y) \sim y^{-1 / 4} e^{-2 i \sqrt{y}}$ near $y=\infty$ (see (4.14)). In order to avoid the root in the exponents we use the following formal function:

$$
\tilde{G}^{ \pm}(v)=\frac{1}{ \pm i} G^{ \pm}\left(v^{2}\right)=\frac{e^{2 v_{ \pm}}}{\sqrt{v_{ \pm}}}\left\{1-\frac{a_{1}}{v_{ \pm}}+\frac{a_{2}}{v_{ \pm}^{2}}-\cdots\right\}
$$

(where $v_{ \pm}= \pm i v$ and $\sqrt{v_{ \pm}}=e^{ \pm i \pi / 4} \sqrt{v}$ for $v>0$ ), which are solutions to the Bessel type equation $\partial_{v} v \partial_{v} \tilde{G}+4 v \tilde{G}=0$, i.e. (4.54) considered in Remark 4.4.

By a sectorial normalization theorem (see [13, 19] and Remark 4.4) the solutions $\tilde{G}^{ \pm}$represent asymptotic series for solutions which are analytic in some sectors about $v=\infty$ (in the complex $v$-plane). In the present case there are two such sectors: $\mathcal{S}_{r}$ (right) and $\mathcal{S}_{l}$ (left) with vertex at $\infty$ of angle $2 \pi-2 \delta(\delta>0$ small $)$ and with the rays $\arg v=0$ and $\arg v=\pi$ as their bisectors (see (4.56)). The latter rays are called the rays of division. Then the sectors $\mathcal{S}_{u}=\mathcal{S}_{r} \cap \mathcal{S}_{l} \cap\{\operatorname{Im} v>0\}$ and $\mathcal{S}_{d}=\mathcal{S}_{r} \cap \mathcal{S}_{l} \cap\{\operatorname{Im} v<0\}$ have angle $\pi-2 \delta$. The sectors $\mathcal{S}_{u}$ and $\mathcal{S}_{d}$ are "transitional" sectors; their bisectors are called the Stokes lines.

We denote by $\tilde{G}_{r}^{ \pm}$and $\tilde{G}_{l}^{ \pm}$the corresponding solutions in the sectors $\mathcal{S}_{r}$ and $\mathcal{S}_{l}$ respectively obtained from the sectorial normalization theorem (see Remark 4.4).

We note the following relations, where $f \prec h$ means that the function $f$ is much smaller than the function $h$ :

$$
\tilde{G}_{r, l}^{+} \prec \tilde{G}_{r, l}^{-} \text {in } \mathcal{S}_{u}, \quad \tilde{G}_{r, l}^{-} \prec \tilde{G}_{r, l}^{+} \text {in } \mathcal{S}_{d} .
$$

The solutions $\tilde{G}_{r}^{ \pm}$(respectively $\tilde{G}_{l}^{ \pm}$) are analytic in the adjacent sectors $\mathcal{S}_{u}$ (up) and $\mathcal{S}_{d}$ (down). Therefore they are expressed as linear combinations of the corresponding solutions $\tilde{G}_{l}^{ \pm}$(respectively $\tilde{G}_{r}^{ \pm}$). The corresponding matrices $C_{u}$ and $C_{d}$ of transformations between the basic solutions are called the Stokes matrices.

Each Stokes matrix is triangular with 1's on the diagonal. We have

$$
C_{u}=\left(\begin{array}{cc}
1 & c_{12} \\
0 & 1
\end{array}\right), \quad C_{d}=\left(\begin{array}{cc}
1 & 0 \\
c_{21} & 1
\end{array}\right)
$$


This means that, after passing from the sector $\mathcal{S}_{r}$ to the sector $\mathcal{S}_{l}$, the basic solutions undergo the following changes:

$$
\begin{array}{lll}
\tilde{G}_{r}^{+}=\tilde{G}_{l}^{+}, & \tilde{G}_{r}^{-}=\tilde{G}_{l}^{-}+c_{12} \tilde{G}_{l}^{+} & \left(\text {in } \mathcal{S}_{u}\right), \\
\tilde{G}_{l}^{-}=\tilde{G}_{r}^{-}, & \tilde{G}_{l}^{+}=\tilde{G}_{r}^{+}+c_{21} \tilde{G}_{l}^{-} & \left(\text {in } \mathcal{S}_{d}\right) .
\end{array}
$$

The rule is that to a given solution one can add a solution with smaller asymptotics at infinity. We shall calculate the coefficients $c_{12}$ and $c_{21}$ using a method from [7], where Stokes matrices associated with the Bessel equation were computed (see also [19]).

We also note the following substitution property of the functions (5.1). Due to the fact that $\sqrt{v_{ \pm}}=e^{ \pm i \pi / 4} \sqrt{v}$ for $v>0$, the independence of the coefficients $a_{j}$ from the sign in (5.1), and the construction in Remark 4.4, we can write

$$
\tilde{G}_{l}^{+}\left(e^{i \pi} v\right)=-\tilde{G}_{r}^{-}(v), \quad \tilde{G}_{l}^{-}\left(e^{i \pi} v\right)=\tilde{G}_{r}^{+}(v), \quad v>0 .
$$

Let $\tilde{G}_{r}^{+}(v)$ on the ray $\arg v=0$ (in the sector $\mathcal{S}_{r}$ ) be represented by the following combination of the basic solutions $\tilde{\Phi}_{1}(v)=\Phi_{2} \circ v^{2}=1-v x^{2}+\cdots$ and $\tilde{\Phi}_{2}(v)=\Phi_{2} \circ v^{2}=\tilde{\Phi}_{1}(v) \cdot \ln v^{2}+\Phi_{3} \circ v^{2}+\cdots$ :

$$
\tilde{G}_{r}^{+}(v)=K_{1} \tilde{\Phi}_{1}(v)+K_{2} \tilde{\Phi}_{2}(v), \quad v>0,
$$

for some coefficients $K_{1}$ and $K_{2}$. After passing to the ray $\arg v=\pi$ (in $\mathcal{S}_{l}$ ) and the substitution $v \mapsto-v$ (using (5.5) and the logarithmic singularity of $\left.\tilde{\Phi}_{2}\right)$ we get

$$
-\tilde{G}_{r}^{-}(v)=\left(K_{1}+2 \pi i K_{2}\right) \tilde{\Phi}_{1}(v)+K_{2} \tilde{\Phi}_{2}(v), \quad v>0 .
$$

Analogously, after passing to the ray $\arg x=2 \pi$ and using an analogue of the relations $(5.5)$, we get

$$
-\tilde{G}_{r}^{+}(v)-c_{21} \tilde{G}_{r}^{-}(v)=\left(K_{1}+4 \pi i K_{2}\right) \tilde{\Phi}_{1}(v)+K_{2} \tilde{\Phi}_{2}(v), \quad v>0 .
$$

Equations (5.6)-(5.8) imply the representation (on $\arg v=0$ )

$$
\tilde{\Phi}_{1}(v)=\frac{i}{2 \pi K_{2}}\left(\tilde{G}_{r}^{+}+\tilde{G}_{r}^{-}\right), \quad \tilde{\Phi}_{2}(v)=\left(\frac{1}{K_{2}}-\frac{i K_{1}}{2 \pi K_{2}^{2}}\right) \tilde{G}^{+}-\frac{i K_{1}}{2 \pi K_{2}^{2}} \tilde{G}^{-},
$$

and the fact that

$$
c_{21}=2 .
$$

Moreover, the asymptotic formula (3.12) implies that $K_{2}=i / \sqrt{\pi}$. Since the solution $\tilde{\Phi}_{2}$ depends on its definition, i.e. modulo $\tilde{\Phi}_{1}$, we find that $\tilde{\Phi}_{2}(v)=$ $-i \sqrt{\pi} \tilde{G}^{+}(v)\left(\bmod \tilde{\Phi}_{1}\right)$ for $v>0$.

In the same way one proves that $c_{12}=-2$ and obtains the representation

$$
\tilde{\Phi}_{1}(v)=\frac{1}{2 \sqrt{\pi}}\left(\tilde{G}_{l}^{-}-\tilde{G}_{l}^{+}\right), \quad \arg v=\pi ;
$$

taking into account the square root of $v$ in $\tilde{G}^{ \pm}$, this agrees with (3.12). We summarize this in the following 
Proposition 5.1. We have $c_{12}=-2$ and $c_{21}=2$ in (5.3). Moreover,

$$
\begin{aligned}
& \tilde{\Phi}_{1}(v)=\frac{1}{2 \sqrt{\pi}}\left(\tilde{G}_{r}^{+}+\tilde{G}_{r}^{-}\right), \quad \tilde{\Phi}_{2}=-i \sqrt{\pi} \cdot \tilde{G}_{r}^{+}\left(\bmod \tilde{\Phi}_{1}\right), \quad \arg v=0 ; \\
& \text { (5.10) } \tilde{\Phi}_{1}(v)=\frac{1}{2 \sqrt{\pi}}\left(\tilde{G}_{l}^{-}-\tilde{G}_{l}^{+}\right), \quad \tilde{\Phi}_{2}=-i \sqrt{\pi} \cdot \tilde{G}_{l}^{+}\left(\bmod \tilde{\Phi}_{1}\right), \quad \arg v=\pi .
\end{aligned}
$$

In (5.9)-(5.10) we give the representation of the function $\tilde{\Phi}(v)$ for $v$ on the two rays of division. But, in fact, these formulas hold true in the whole sector $\mathcal{S}_{r, l}$ which contains the corresponding ray of division. The same remark applies in other expansions which are given below.

Calculation of the Stokes matrices associated with the Bessel type equation (1.13) runs practically in the same way as in the proof of Proposition 5.1. One has the formal solution $H^{ \pm}(z) \sim z^{1 / 4} e^{\mp 2 i \sqrt{z}}$ (see (4.15)). After the transformation $z=w^{2}$ we can define the following functions:

$$
\tilde{H}^{ \pm}(w)=\sqrt{w_{ \pm}} e^{-2 w_{ \pm}}\left\{1-\frac{b_{1}}{w_{ \pm}}+\frac{b_{2}}{w_{ \pm}^{2}}-\cdots\right\} .
$$

They satisfy the Bessel type equation (4.56) with another pair of solutions

$$
\tilde{\Theta}_{1}(w)=w-\frac{1}{2} w^{2}+\cdots, \quad \tilde{\Theta}_{2}(w)=\tilde{\Theta}_{1}(w) \cdot \ln w+\tilde{\Theta}_{3}(w)
$$

(with analytic $\tilde{\Theta}_{1}$ and $\tilde{\Theta}_{3}$ ).

Now we have the same sectors $\mathcal{S}_{r, l}$, with analytic solutions $\tilde{H}_{r, l}^{ \pm}$, and $\mathcal{S}_{u, d}$ about $w=\infty$, but with domination relations different than in (5.2). Therefore the corresponding Stokes matrices take the form

$$
D_{u}=\left(\begin{array}{cc}
1 & 0 \\
d_{21} & 1
\end{array}\right), \quad D_{d}=\left(\begin{array}{cc}
1 & d_{12} \\
0 & 1
\end{array}\right) .
$$

Anyway (using also (3.15) and (3.16)) we arrive at the following result, where $(5.17)$ is a consequence of the factor $\sqrt{w_{ \pm}}$in the definition of $\tilde{H}^{ \pm}$ $\left(\tilde{H}_{l}^{ \pm}\left(e^{2 \pi i} w\right)=-\tilde{H}_{l}^{ \pm}(w)\right)$.

Proposition 5.2. We have $d_{12}=-2$ and $d_{21}=2$ in (5.12). Moreover,

$$
\begin{aligned}
\tilde{\Theta}_{1}(w) & =\frac{1}{2 i \sqrt{\pi}}\left(\tilde{H}_{r}^{-}-\tilde{H}_{r}^{+}\right), \\
\tilde{\Theta}_{2}(v) & =-\sqrt{\pi} \cdot \tilde{H}_{r}^{+}\left(\bmod \tilde{\Theta}_{1}\right), \quad \arg w=0 ; \\
\tilde{\Theta}_{1}(w) & =\frac{-1}{2 i \sqrt{\pi}}\left(\tilde{H}_{l}^{-}+\tilde{H}_{l}^{+}\right), \\
\tilde{\Theta}_{2}(v) & =-\sqrt{\pi} \cdot \tilde{H}_{l}^{-}\left(\bmod \tilde{\Theta}_{1}\right), \quad \arg w=\pi .
\end{aligned}
$$


In particular, we get

$$
\begin{array}{ll}
\tilde{H}_{r}^{+}(w)=\tilde{H}_{r}^{-}(w)=(-1 / \sqrt{\pi}) \cdot \tilde{\Theta}_{2}\left(\bmod \tilde{\Theta}_{1}\right), & \arg w=0 \\
\tilde{H}_{l}^{+}(w)=-\tilde{H}_{l}^{-}(w)=(1 / \sqrt{\pi}) \cdot \tilde{\Theta}_{2}\left(\bmod \tilde{\Theta}_{1}\right), & \arg w=\pi \\
\tilde{H}_{l}^{+}(w)=-\tilde{H}_{l}^{-}(w)=(-1 / \sqrt{\pi}) \cdot \tilde{\Theta}_{2}\left(\bmod \tilde{\Theta}_{1}\right), & \arg w=-\pi
\end{array}
$$

5.2. Stokes operators for the hypergeometric equation. This subsection is the core of the whole paper. Here we prove that the connection coefficient $B(x)$ from the equation $\varphi_{1}=A(x) \theta_{1}+B(x) \theta_{2}$ (see (1.9)), which is related to the generating function $f_{2}(x)$, admits for large $x$ a representation via WKB type functions which are subject to the Stokes phenomenon which is trivial.

Recall that the hypergeometric equation (1.6) is equivalent to the Bessel type equation (1.12) near the point $t=0$ and to the Bessel type equation (1.13) near the point $t=1$ (compare Proposition 3.1). Application of the matrix $\mathcal{H}_{0}$ (which realizes the equivalence near $t=0$ ) from the right to the row vector $\left(\tilde{G}_{r}^{+}(x \sqrt{t}), \tilde{G}_{r}^{-}(x \sqrt{t})\right)$ gives two row vectors of WKB solutions

$$
\begin{aligned}
& \left(M_{r}^{+}(x) g_{u}^{+}, M_{r}^{-}(x) g_{u}^{-}+M_{r}(x) g_{u}^{+}\right), \\
& \left(N_{r}^{+}(x) g_{d}^{+}+N_{r}(x) g_{d}^{-}, N_{r}^{-}(x) g_{d}^{-}\right) .
\end{aligned}
$$

Here $g_{u, d}^{ \pm}$are the WKB solutions from Proposition 4.1 and $M(x), M_{+}(x)$, $M_{-}(x), N(x), N_{+}(x), N_{-}(x)$ are functions of $x$ holomorphic in suitable domains.

Formula (5.18) holds in the domain

$$
\mathcal{D}_{u} \cap\left\{x \sqrt{t} \in \mathcal{S}_{r}\right\} \cap\{|t|<\gamma\},
$$

where $\mathcal{D}_{u}$ is defined in (4.49) (recall that in $\mathcal{D}_{u}$ the parameter $t$ is in $\mathcal{W}$ and is close to the segment $(0,1))$ and $\gamma$ is a small constant, but $\gamma>\varepsilon$ where $\varepsilon$ appears in the definition of $\mathcal{W}$. Note that the domain (5.20) contains points $(t, x)$ with $\operatorname{Im} x \geq 0$ (which is clear) but also with $\operatorname{Im} x<0$ (although small); this can be seen from the definitions of $\mathcal{D}_{u}$ in (4.49) and of $\mathcal{S}_{r}$ in (4.55).

The functions $M_{r}, M_{r}^{ \pm}$are analytic in the domain (5.20). But they are functions of $x$ only. So they are analytic in a rather large domain $\left\{\operatorname{Im} x>-\alpha^{\prime},|x|>1 / \varepsilon\right\}$ for some $\alpha^{\prime}>0$.

Analogously, (5.19) holds in the domain

$$
\mathcal{D}_{d} \cap\left\{x \sqrt{t} \in \mathcal{S}_{r}\right\} \cap\left\{\gamma_{1}<|t|<\gamma_{2}\right\}
$$

and the functions $N_{r}, N_{r}^{ \pm}$are analytic in the domain $\left\{\operatorname{Im} x<\alpha^{\prime},|x|>1 / \varepsilon\right\}$. 
Application (from the right) of the matrix $\mathcal{H}_{0}$ to the vector $\left(\tilde{G}_{l}^{+}(x \sqrt{t})\right.$, $\left.\tilde{G}_{l}^{-}(x \sqrt{t})\right)$ gives the following analogue of (5.18)-(5.19):

$$
\begin{aligned}
& \left(M_{l}^{+}(x) g_{u}^{+}, M_{l}^{-}(x) g_{u}^{-}+M_{l}(x) g_{u}^{+}\right), \\
& \left(N_{l}^{+}(x) g_{d}^{+}+N_{l}(x) g_{d}^{-}, N_{l}^{-}(x) g_{d}^{-}\right),
\end{aligned}
$$

which hold in respective domains analogous to the domains (5.20) and (5.21) (where $\mathcal{S}_{r}$ is replaced with $\mathcal{S}_{l}$ ). Here also the functions $M_{l}, M_{l}^{ \pm}$are analytic in the domain $\left\{\operatorname{Im} x>-\alpha^{\prime},|x|>1 / \varepsilon\right\}$ and the functions $N_{r}, N_{r}^{ \pm}$are analytic in the domain $\left\{\operatorname{Im} x<\alpha^{\prime},|x|>1 / \varepsilon\right\}$.

The Stokes phenomenon for the solutions $\tilde{G}_{r, l}^{ \pm}$means some relations between their images under $\mathcal{H}_{0}$. From Proposition 5.1 and equations (5.18) and (5.22) we get $M_{r}^{+} g_{u}^{+}=M_{l}^{+} g_{u}^{+}$and $M_{r}^{-} g_{u}^{-}+M_{r} g_{u}^{+}=M_{l}^{-} g_{u}^{-}+M_{l} g^{+}-$ $2\left(M_{l}^{+} g_{u}^{+}\right)$, i.e.

$$
M_{r}^{+}=M_{l}^{+}, \quad M_{r}^{-}=M_{l}^{-}, \quad M_{r}=M_{l}-2 M_{l}^{+} .
$$

Similarly (5.19) and (5.23) give

$$
N_{r}^{+}=N_{l}^{+}, \quad N_{r}^{-}=N_{l}^{-}, \quad N_{r}=N_{l}-2 N_{l}^{-} .
$$

Moreover, the matrix $\mathcal{H}_{0}\left(t, x^{-1}\right)$ is invariant with respect to conjugation (it has real "coefficients"). Also the solutions $\tilde{G}_{r, l}^{ \pm}$and $g_{u, d}^{ \pm}$have some conjugation symmetry properties. For instance, $\overline{\tilde{G}_{r}^{+}(v)}=\tilde{G}_{r}^{-}(\bar{v})$ and analogous properties for $g_{u, d}^{ \pm}$are given in (4.52). This implies additionally to (5.24) and (5.25) the relations

$$
\overline{M_{r}^{+}(x)}=N_{r}^{-}(\bar{x}), \quad \overline{M_{r}^{-}(x)}=N_{r}^{-}(\bar{x}), \quad \overline{M(x)}=N(\bar{x}) .
$$

Finally, the normalization condition $2 \sqrt{\pi} \varphi_{1}=g_{u}^{+}+g_{u}^{-}=g_{d}^{+}+g_{d}^{-}$for $t, x>0$ (see (4.53)) means that

$$
M_{r}^{-}=1, \quad M_{r}^{+}+M_{r}=1, \quad N_{r}^{+}=1, \quad N_{r}^{-}+N_{r}=1 .
$$

It follows that there remains only one "free" coefficient, which can be chosen as $M_{r}^{+}(x)$.

Now we analogously calculate the relations between the WKB solutions $\tilde{H}_{r, l}^{ \pm}=\tilde{H}_{r, l}^{ \pm}(x \sqrt{s})$ and

$$
h_{u, d}^{ \pm}= \pm i x e^{\mp i \pi x} g_{u, d}^{ \pm}(1-s ; x)
$$

(compare (4.57) in Definition 4.3). Recall that $h_{u}^{-} \prec h_{u}^{+}$in $\mathcal{D}_{u}$ and $h_{d}^{+} \prec h_{d}^{-}$ in $\mathcal{D}_{d}$. The (right) action of the matrix $\mathcal{H}_{1}^{-1}$ from Proposition 3.1 on the WKB solutions (5.28) is the following: 


$$
\begin{aligned}
& h_{u}^{+} \mapsto m_{r}^{+}(x) \tilde{H}_{r}^{+}+m_{r}(x) \tilde{H}_{r}^{-}=m_{l}^{+}(x) \tilde{H}_{l}^{+}+m_{l}(x) \tilde{H}_{l}^{-}, \\
& h_{u}^{-} \mapsto m_{r}^{-}(x) \tilde{H}_{r}^{-}=m_{l}^{-} \tilde{H}_{l}^{-}, \\
& h_{d}^{+} \mapsto n_{r}^{+}(x) \tilde{H}_{r}^{+}=n_{l}^{+}(x) \tilde{H}_{l}^{+}, \\
& h_{d}^{-} \mapsto n_{r}^{-}(x) \tilde{H}_{r}^{-}+n_{r}(x) \tilde{H}_{r}^{+}=n_{l}^{-} \tilde{H}_{l}^{-}+n_{l}(x) \tilde{H}_{l}^{+} .
\end{aligned}
$$

Here the coefficients are analytic in the corresponding domains $\{ \pm \operatorname{Im} x>$ $\left.-\alpha^{\prime},|x|>1 / \varepsilon\right\}$ and satisfy the relations

$$
\begin{aligned}
m_{r}^{+} & =m_{l}^{+}, \quad m_{r}^{-}=m_{l}^{-}, \quad m_{l}=m_{r}+2 m_{r}^{+}, \\
n_{r}^{+} & =n_{l}^{+}, \quad n_{r}^{-}=n_{l}^{-}, \quad n_{l}=n_{r}+2 n_{r}^{-}, \\
\overline{m_{r}^{+}(x)} & =n_{r}^{-}(\bar{x}), \quad \overline{m_{r}^{-}(x)}=n^{+}(\bar{x}), \quad \overline{m(x)}=n(\bar{x}) .
\end{aligned}
$$

The latter relations mean that the coefficients are analytic and single-valued functions in the whole neighborhood of $x=\infty$. But at this moment we cannot claim normalization relations like those in (5.27).

But we can now express the solution $\varphi_{1}=2\left(g_{u}^{+}+g_{u}^{-}\right) / \sqrt{\pi}=2\left(g_{d}^{+}+\right.$ $\left.g_{d}^{-}\right) / \sqrt{\pi}$ as $B(x) \theta_{1}\left(\bmod \theta_{1}\right)$. By Propositions 4.1 and 5.2 and $(5.28)-(5.29)$ we have

$$
\begin{aligned}
\varphi_{1} & =\frac{1}{2 \sqrt{\pi}}\left(g_{u}^{+}+g_{u}^{-}\right)=\frac{1}{2 i x \sqrt{\pi}}\left\{e^{i \pi x} h_{u}^{+}-e^{-i \pi x} h_{u}^{-}\right\} \\
& \mapsto \frac{1}{2 i x \sqrt{\pi}}\left\{e^{i \pi x}\left(m_{r}^{+}(x) \tilde{H}_{r}^{+}+m_{r}(x) \tilde{H}_{r}^{-}\right)-e^{-i \pi x} m_{r}^{-}(x) \tilde{H}_{r}^{-}\right\} \\
& =\frac{-1}{2 \pi i x}\left\{e^{i \pi x}\left(m_{r}^{+}(x)+m_{r}(x)\right)-e^{-i \pi x} m_{r}^{-}(x)\right\} \cdot \tilde{\Theta}_{2}\left(\bmod \tilde{\Theta}_{1}\right) \\
& \mapsto \frac{-1}{2 \pi i x}\left\{e^{i \pi x}\left(m_{r}^{+}(x)+m_{r}(x)\right)-e^{-i \pi x} m_{r}^{-}(x)\right\} \cdot \theta_{2}\left(\bmod \theta_{1}\right),
\end{aligned}
$$

where the first arrow denotes the map defined by $\mathcal{H}_{1}^{-1}$ and the second arrow corresponds to the matrix $\mathcal{H}_{1}$. Analogously we find the following relations:

$$
\begin{aligned}
& \varphi_{1}=\frac{-1}{2 \pi i x}\left\{e^{i \pi x}\left(-m_{l}^{+}(x)+m_{l}(x)\right)-e^{-i \pi x} m_{l}^{-}(x)\right\} \cdot \theta_{2}\left(\bmod \theta_{1}\right), \\
& \varphi_{1}=\frac{-1}{2 \pi i x}\left\{e^{i \pi x} n_{r}^{+}(x)-e^{-i \pi x}\left(n_{r}^{-}(x)+n_{r}(x)\right)\right\} \cdot \theta_{2}\left(\bmod \theta_{1}\right), \\
& \varphi_{1}=\frac{-1}{2 \pi i x}\left\{e^{i \pi x} n_{l}^{+}(x)-e^{-i \pi x}\left(-n_{l}^{-}(x)+n_{l}(x)\right)\right\} \cdot \theta_{2}\left(\bmod \theta_{1}\right) .
\end{aligned}
$$

Subtracting the right hand side of (5.32) from the right hand side of (5.31) we get $-1 / 2 \pi i x$ times

$$
e^{i \pi x}\left\{m_{r}^{+}(x)+m_{l}^{+}(x)+m_{r}(x)-m_{l}(x)\right\}-e^{-i \pi x}\left(m_{r}^{-}(x)-m_{l}^{-}\right)
$$

times $\theta_{2}\left(\bmod \theta_{1}\right)$; this is zero due to (5.30). The same argument shows that the right hand sides of (5.33)-(5.34) coincide on the intersection of their domains. 
The above implies that the connection coefficient $B(x)$ is represented via the WKB type functions

$$
F^{ \pm}(x)=\frac{\exp ( \pm i \pi x)}{x} \cdot \omega^{ \pm}\left(x^{-1}\right)
$$

which are subject to the trivial Stokes phenomenon in the sense that the functions $\omega^{ \pm}\left(x^{-1}\right)$ are analytic and single-valued near $x^{-1}=0$. We regard this as the fundamental result of the paper.

THEOREM 5.1. The generating function (1.3) for multiple zeta values $\zeta(2, \ldots, 2)$ has a representation

$$
f_{2}(x)=F^{+}(x)+F^{-}(x), \quad x \rightarrow \infty,
$$

where $F^{ \pm}(x)$ are single-valued functions of the form (5.35). This implies that $f_{2}(s)=\sin \pi x / \pi x$.

Proof. The first statement of the theorem was proved above. To prove the second statement we note that the function $f_{2}(x)$ vanishes at the points $x= \pm 1, \pm 2, \ldots$. Since the function $\sin \pi x / x$ has simple zeroes at those points, we find that the function

$$
f_{2}(x) /(\sin \pi x / x)
$$

is entire on $\mathbb{C}$. By the first part of Theorem 5.1 it is bounded at infinity. Therefore it is a constant function equal to $1 / \pi$, since $f(0)=1$.

REMARK 5.1. Potential importance of this theorem relies upon the fact that in its proof we do not use Proposition 2.1 about the fundamental relation between solutions near $t=0$ and $t=1$.

Moreover, the coefficients in the expansions of $\omega^{ \pm}$from (5.35) in powers of $x^{-1}$ can be derived without knowing the function $f_{2}(x)$. Namely, first we use the formal expansion of $\varphi_{1}$ from Lemma 4.4 (via the testing WKB solutions $g_{0}^{ \pm}$and the series $C\left(x^{-2}\right)$ ), next we express the solutions $g_{0}^{ \pm}$via the WKB solutions $h_{0}^{ \pm}$from Definition 4.3 and, finally, using the integral formulas for $\theta_{1}$ and $\hat{\theta}_{2}$ and the stationary phase formula we are able to rewrite $h_{0}^{ \pm}$in terms of $\theta_{1}, \hat{\theta}_{2}$. Also the triviality of the Stokes phenomenon can be deduced at the formal level in this way.

We hope that the approach to the hypergeometric equation (1.6) developed in this paper is novel and can be applied to other hypergeometric type equations of the form (1.5).

REMARK 5.2. One could consider the second order differential equation satisfied by the summands $F^{+}(x)$ and $F^{-}(x)$, i.e.

$$
\operatorname{det}\left[\begin{array}{ccc}
f & \partial_{x} f & \partial_{x}^{2} f \\
F^{+} & \partial_{x} F^{+} & \partial_{x}^{2} F^{+} \\
F^{-} & \partial_{x} F^{-} & \partial_{x}^{2} F^{-}
\end{array}\right]=0 .
$$


By definition it is an equation defined near $x=\infty$, with meromorphic and irregular singularity at infinity. But when one assumes that it extends to a Bessel type equation in $\mathbb{C}$ with regular singularity at $x=0$, then one arrives at the equation

$$
\partial_{x}^{2} f+(2 / x) \partial_{x} f+\pi^{2} f=0
$$

with the solutions $\sin \pi x / x$ and $\cos \pi x / x$.

Unfortunately, it seems that this argument fails in the case of higher order hypergeometric type equations, like (1.18).

Acknowledgments. We would like to thank Grzegorz Eysik for his careful reading of an initial version of the work and for his remarks.

This research was supported by Polish MNiSzW Grants No N N201 271035, 1 P03A 01529 and N N201 397937.

\section{References}

[1] H. Bateman et al., Higher Transcendental Functions, Vol. 1, McGraw-Hill, New York, 1953.

[2] -, Higher Transcendental Functions, Vol. 2, McGraw-Hill, New York, 1953.

[3] G. D. Birkhoff, On the asymptotic character of the solutions of certain differential equations containing a parameter, Trans. Amer. Math. Soc. 9 (1908), 219-230.

[4] L. Brillouin, La mécanique ondulatoire de Schrödinger: une méthode générale de résolution par approximations successives, C. R. Acad. Sci. Paris 183 (1926), 24-26.

[5] M. B. Fedoryuk, Asymptotic Analysis. Linear Ordinary Differential Equations, Springer, Berlin, 1993 [Russian original: Nauka, Moscow, 1983].

[6] A. Gray and G. B. Mathews, A Treatise on Bessel Functions and Their Applications to Physics, McMillan, London, 1931.

[7] J. M. A. Heading, An Introduction to Phase-Integral Methods, Wiley, New York, 1977.

[8] Hoang Ngoc Minh, M. Petitot and J. van der Hoeven, Shuffle algebra and polylogarithms, in: Formal Power Series and Algebraic Combinatorics (Toronto, 1998), Discrete Mah. 225 (2000), 217-230.

[9] M. Kontsevich and D. Zagier, Periods, in: Mathematics Unlimited-2001 and beyond, Springer, Berlin, 2001, 771-808.

[10] H. A. Kramers, Wellenmechanik und halbzählige Quantisierung, Z. Physik 39 (1926), 828-840.

[11] L. Schiff, Quantum Mechanics, McGraw-Hill, New York, 1968.

[12] G. G. Stokes, On the discontinuity of arbitrary constants that appear as multipliers of semi-convergent series, Acta Math. 26 (1902), 393-397.

[13] W. Wasow, Asymptotic Expansions for Ordinary Differential Equations, Wiley, New York, 1965.

[14] G. Wentzel, Eine Verallgemeinerung der Quantenbedingungen für die Zwecke der Wellenmechanik, Z. Physik 38 (1926), 518-529.

[15] D. Zagier, Values of zeta functions and their applications, in: First European Congress of Mathematicians, Vol. 2, Progr. Math. 120, Birkhäuser, Basel, 1994, 497-512.

[16] M. Zakrzewski and H. Żołądek, Linear differential equations and multiple zeta values. II. A generalization of the WKB method, preprint, Univ. of Warsaw, 2010. 
[17] M. Zakrzewski and H. Żołądek, Linear differential equations and multiple zeta values. III. Zeta(3), in preparation.

[18] H. Żołądek, Note on multiple zeta-values, Bul. Acad. Ştiinţe Rep. Moldov. Mat. 2003, no. 1 (41), 78-82.

[19] —, The Monodromy Group, Birkhäuser, Basel, 2006.

Michał Zakrzewski, Henryk Żołądek

Institute of Mathematics

University of Warsaw

Banacha 2

02-097 Warszawa, Poland

E-mail: zakrzewski@mimuw.edu.pl

zoladek@mimuw.edu.pl

Received 3 March 2008;

in revised form 15 June 2010 